\title{
Higher-spin Cotton tensors and massive gauge-invariant actions in $\mathrm{AdS}_{3}$
}

\section{Sergei M. Kuzenko and Michael Ponds}

Department of Physics M013, The University of Western Australia, 35 Stirling Highway, Perth, WA 6009, Australia

E-mail: sergei.kuzenko@uwa.edu.au, michael.ponds@research.uwa.edu.au

ABSTRACT: In a conformally flat three-dimensional spacetime, the linearised higher-spin Cotton tensor $\mathfrak{C}_{\alpha(n)}(h)$ is the unique conserved conformal current which is a gauge-invariant descendant of the conformal gauge prepotential $h_{\alpha(n)}$. The explicit form of $\mathfrak{C}_{\alpha(n)}(h)$ is well known in Minkowski space. Here we solve the problem of extending the Minkowskian result to the case of anti-de Sitter (AdS) space and derive a closed-form expression for $\mathfrak{C}_{\alpha(n)}(h)$ in terms of the AdS Lorentz covariant derivatives. It is shown that every conformal higherspin action $S_{\mathrm{CS}}^{(n)}[h] \propto \int \mathrm{d}^{3} x e h^{\alpha(n)} \mathfrak{C}_{\alpha(n)}(h)$ factorises into a product of $(n-1)$ first-order operators that are associated with the spin- $n / 2$ partially massless AdS values. Our findings greatly facilitate the on-shell analysis of massive higher-spin gauge-invariant actions in $\mathrm{AdS}_{3}$. The main results are extended to the case of $\mathcal{N}=1$ AdS supersymmetry. In particular, we derive simple expressions for the higher-spin super-Cotton tensors in $\mathrm{AdS}_{3}$.

KEYwords: Higher Spin Gravity, Supergravity Models, Superspaces

ArXiv EPrint: 2103.11673 


\section{Contents}

1 Introduction 1

2 Higher-spin Cotton tensors and on-shell fields in $\mathrm{AdS}_{3} \quad 5$

$2.1 \mathrm{AdS}_{3}$ geometry 5

$\begin{array}{lll}2.2 & \text { On-shell massive and partially massless fields } & 6\end{array}$

2.3 Linearised higher-spin Cotton tensors 8

2.4 Factorisation of the Cotton tensors and CHS action 9

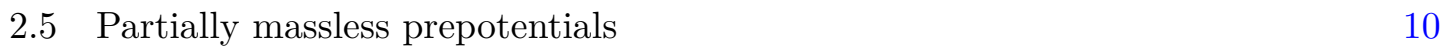

3 Massive higher-spin gauge models $\quad 11$

$\begin{array}{lll}3.1 & \text { New topologically massive higher-spin gauge models } & 11\end{array}$

$\begin{array}{ll}3.2 & \text { Topologically massive higher-spin gauge models } \\ \end{array}$

4 Supersymmetric higher-spin gauge models in $\mathrm{AdS}_{3} \quad 16$

$\begin{array}{lll}4.1 & \text { Conformal supergravity and super-Cotton tensors } & 16\end{array}$

$\begin{array}{lll}4.2 & \text { Higher-spin super-Cotton tensors in conformally flat superspace } & 17\end{array}$

$\begin{array}{ll}4.3 \mathcal{N}=1 \text { AdS superspace geometry } & 19\end{array}$

4.4 On-shell massive and partially massless superfields in $\mathrm{AdS}_{3} \quad 20$

$\begin{array}{ll}4.5 & \text { Component analysis } \\ & 22\end{array}$

4.6 Linearised higher-spin super-Cotton tensors 23

4.7 Factorisation of the higher-spin super-Cotton tensor 25

$\begin{array}{ll}4.8 \text { Massive } \mathcal{N}=1 \text { gauge actions } & 26\end{array}$

$\begin{array}{lll}5 & \text { Discussion } & 27\end{array}$

A Two-component spinor toolkit $\quad 28$

$\begin{array}{ll}\text { B Generating function formalism } & 30\end{array}$

$\begin{array}{ll}\text { C Gauge completeness } & 31\end{array}$

\section{Introduction}

The conformal geometry of spacetime in four and higher dimensions is controlled by the Weyl tensor. The necessary and sufficient condition for spacetime to be conformally flat is that the Weyl tensor is equal to zero, see e.g. [1]. If conformal gravity is realised as the gauge theory of the conformal group [2,3], then the algebra of conformally covariant derivatives is determined by the Weyl tensor and its covariant derivatives [4-6]. The conformal connection becomes flat if the Weyl tensor vanishes. 
In three dimensions, the Weyl tensor is identically zero and all information about conformal geometry is encoded in the Cotton tensor, $C_{a b c}=-C_{b a c}$. Spacetime is conformally flat if and only if the Cotton tensor vanishes [1] (see [5] for a modern proof). The commutator of two conformally covariant derivatives $\nabla_{a}$ involves only the Cotton tensor [5] (see also [7])

$$
\left[\nabla_{a}, \nabla_{b}\right]=\frac{1}{2} C_{a b c} K^{c}, \quad C_{a b c}=-\varepsilon_{a b d} C^{d}{ }_{c},
$$

where $K^{c}$ is the special conformal generator. The algebraic structure of the Cotton tensor is described by the relations

$$
C_{a b}=C_{b a}, \quad C^{b}{ }_{b}=0 .
$$

The Cotton tensor is a primary field, $K^{c} C_{a b}=0$, of dimension 3 and obeys the Bianchi identity

$$
\nabla^{b} C_{b a}=0 .
$$

As follows from (1.1a), the conformal connection becomes flat if the Cotton tensor vanishes.

Since the pioneering work by Fradkin and Tseytlin [8], there has been much interest in conformal higher-spin (CHS) theories in diverse dimensions, see [9-18] for an incomplete list of works published within a quarter-century after [8]. This interest has truly exploded in the last decade (see, e.g., [19-31] and references therein), and some comments on the literature will be given below. In the case of three dimensions, higher-spin generalisations of the Cotton tensor are of central importance and lie at the heart of many recent studies [3244]. Linearised higher-spin Cotton tensors in $\mathrm{AdS}_{3}$ will be the main object of interest in this work.

In Minkowski space, linearised higher-spin extensions of the Cotton tensor were constructed in the bosonic [10] and fermionic [39] cases. An alternative derivation of the higher-spin Cotton tensors was given in [36, 43], where the latter played an integral role in establishing a conformal geometry of higher-spin gauge fields. Supersymmetric extensions of the higher-spin Cotton tensors were derived in [39,40] for $\mathcal{N}=1$, in [38] for $\mathcal{N}=2$ and in [44] for $\mathcal{N}>2$ Poincaré supersymmetry. It was argued in $[7,42]$ that higher-spin Cotton tensors exist on any conformally flat background, although explicit expressions for them in terms of Lorentz covariant derivatives and conformal prepotentials are difficult to derive if the spacetime curvature is non-vanishing. It is pertinent here to elaborate on these points in some more detail.

Given a conformally flat spacetime $\mathcal{M}^{3}$, its geometry may be described in terms of torsion-free Lorentz covariant derivatives

$$
\mathcal{D}_{a}=e_{a}+\omega_{a}=e_{a}{ }^{m} \partial_{m}+\frac{1}{2} \omega_{a}^{b c} M_{b c}, \quad\left[\mathcal{D}_{a}, \mathcal{D}_{b}\right]=\frac{1}{2} R_{a b}{ }^{c d} M_{c d},
$$

where $e_{a}{ }^{m}$ is the inverse vielbein, $\omega_{a}^{b c}$ the Lorentz connection, and $M_{a b}=-M_{b a}$ the Lorentz generators. Since $\mathcal{M}^{3}$ is conformally flat, its Cotton tensor $C_{a b c}$ is zero,

$$
C_{a b c}:=\mathcal{D}_{a} P_{b c}-\mathcal{D}_{b} P_{a c}=0, \quad P_{a b}=R_{a b}-\frac{1}{4} \eta_{a b} R,
$$


where $P_{a b}$ denotes the Schouten tensor. Let $\zeta=\zeta^{m} \partial_{m}=\zeta^{a} e_{a}$ be a conformal Killing vector field on $\mathcal{M}^{3}$,

$$
\mathcal{D}^{a} \zeta^{b}+\mathcal{D}^{b} \zeta^{a}=2 \eta^{a b} \sigma[\zeta], \quad \sigma[\zeta]=\frac{1}{3} \mathcal{D}_{b} \zeta^{b}
$$

The conformal Killing vector fields span the conformal algebra of $\mathcal{M}^{3}$, which is isomorphic to $\mathfrak{s o}(3,2)$. A primary tensor field $\varphi$ (with suppressed indices) possesses the following conformal transformation law

$$
-\delta_{\zeta} \varphi=\left(\zeta^{b} \mathcal{D}_{b}+\frac{1}{2} K^{b c}[\zeta] M_{b c}+d_{\varphi} \sigma[\zeta]\right) \varphi, \quad K^{b c}[\zeta]=\frac{1}{2}\left(\mathcal{D}^{b} \zeta^{c}-\mathcal{D}^{c} \zeta^{b}\right)
$$

where $d_{\varphi}$ is the dimension of $\varphi$.

Let us fix a positive integer $n \geq 2$. In the two-component spinor notation, the conformal gauge field $h_{\alpha(n)}:=h_{\alpha_{1} \ldots \alpha_{n}}=h_{\left(\alpha_{1} \ldots \alpha_{n}\right)}$ is a real primary field of dimension

$$
d_{h_{\alpha(n)}}=2-\frac{n}{2}
$$

with the gauge transformation law ${ }^{1}$

$$
\delta_{\xi} h_{\alpha(n)}=\mathcal{D}_{\alpha(2)} \xi_{\alpha(n-2)} .
$$

The Cotton tensor $\mathfrak{C}_{\alpha(n)}(h)$ associated with $h_{\alpha(n)}$ is defined to satisfy the following three conditions: (i) it is a primary descendent of $h_{\alpha(n)}$; (ii) it is transverse (or covariantly conserved),

$$
0=\mathcal{D}^{\beta(2)} \mathfrak{C}_{\beta(2) \alpha(n-2)}(h)
$$

(iii) it is invariant under the gauge transformations (1.7),

$$
0=\mathfrak{C}_{\alpha(n)}\left(\delta_{\xi} h\right)
$$

The conditions (i) and (ii) fix the dimension of $\mathfrak{C}_{\alpha(n)}(h)$ to be

$$
d_{\mathfrak{C}_{\alpha(n)}}=1+\frac{n}{2}
$$

The Cotton tensor $\mathfrak{C}_{\alpha(n)}(h)$ proves to exist, and is unique modulo an overall normalisation. The reader can ask the natural question: what goes wrong if the background manifold is not conformally flat? The answer is that the properties (1.8) and (1.9) break down for $n>2$,

$$
C_{a b c} \neq 0 \quad \Longrightarrow \quad \mathcal{D}^{\beta(2)} \mathfrak{C}_{\beta(2) \alpha(n-2)}(h)=\mathcal{O}(C), \quad \mathfrak{C}_{\alpha(n)}\left(\delta_{\xi} h\right)=\mathcal{O}(C) .
$$

We refer the reader to $[7,42]$ for the technical details.

\footnotetext{
${ }^{1}$ Here, and often below, we make use of the compact notational convention introduced by Vasiliev [45]: $V_{\alpha(n)} U_{\alpha(m)}=V_{\left(\alpha_{1} \ldots \alpha_{n}\right.} U_{\left.\alpha_{n+1} \ldots \alpha_{n+m}\right)}$.
} 
In Minkowski space, the covariant derivative can be chosen to coincide with a partial one, $\mathcal{D}_{a}=\delta_{a}{ }^{m} \partial_{m}=\partial_{a}$. Then one can derive the following closed-form expression for the higher-spin Cotton tensor ${ }^{2}[39]$

$$
\mathfrak{C}_{\alpha(n)}(h):=\frac{1}{2^{n-1}} \sum_{j=0}^{\lceil n / 2\rceil-1}\left(\begin{array}{c}
n \\
2 j+1
\end{array}\right) \square^{j}\left(\partial_{\alpha}^{\beta}\right)^{n-2 j-1} h_{\alpha(2 j+1) \beta(n-2 j-1)} .
$$

In the bosonic case, $n=2 s$, where $s \geq 1$ is an integer, the above expression is equivalent to those given in $[10,36]$. It should be pointed out that the conformal spin- 3 case, $n=6$, was studied for the first time in [46]. The spin-3/2 case, $n=3$, was considered in [47]. The field strength $\mathfrak{C}_{\alpha(3)}(h)$ is the linearised version of the Cottino vector-spinor [48, 49].

The extension of (1.12) to a conformally flat spacetime $\mathcal{M}^{3}$ is obtained [7] by replacing $\partial_{a} \rightarrow \nabla_{a}$. Since the commutator (1.1a) vanishes in every conformally flat spacetime, all the properties of $\mathfrak{C}_{\alpha(n)}(h)$ follow through. However, in addition to the Lorentz generators, $\nabla_{a}$ involves the special conformal generators $K_{b}$ (in a special gauge where the dilatation connection is gauged away). In order to switch from the description in terms of $\nabla_{a}$ to that of the standard Lorentz covariant derivative $\mathcal{D}_{a}$, one has to go through the process of degauging $[5,7]$. This includes expanding $\nabla_{a}$ using the relation

$$
\nabla_{a}=\mathcal{D}_{a}+\frac{1}{2} P_{a}^{b} K_{b}
$$

and then evaluating the action of all special conformal generators. The description of $\mathfrak{C}_{\alpha(n)}(h)$ in terms of $\nabla_{a}$ suffices to describe the conformal higher-spin action (2.23). However, in order to formulate non-conformal massive actions such as (3.1), one has to express $\mathfrak{C}_{\alpha(n)}(h)$ in terms of the Lorentz covariant derivatives $\mathcal{D}_{a}$.

It turns out that, even in the case of a maximally symmetric spacetime, the implementation of the degauging procedure for the higher-spin Cotton tensor is extremely non-trivial. For example, in $\mathrm{AdS}_{3}$ the expressions for $\mathfrak{C}_{\alpha(n)}(h)$ with $3 \leq n \leq 6$ are given by [42]

$$
\begin{aligned}
\mathfrak{C}_{\alpha(3)}(h)= & \frac{1}{2^{2}}\left(3\left(\mathcal{D}_{\alpha}{ }^{\beta}\right)^{2} h_{\alpha \beta(2)}+\left(\mathcal{Q}-9 \mathcal{S}^{2}\right) h_{\alpha(3)}\right) \\
\mathfrak{C}_{\alpha(4)}(h)= & \frac{1}{2^{3}}\left(4\left(\mathcal{D}_{\alpha}{ }^{3}\right)^{3} h_{\alpha \beta(3)}+4\left(\mathcal{Q}-20 \mathcal{S}^{2}\right) \mathcal{D}_{\alpha}{ }^{\beta} h_{\alpha(3) \beta}\right) \\
\mathfrak{C}_{\alpha(5)}(h)= & \frac{1}{2^{4}}\left(5\left(\mathcal{D}_{\alpha}{ }^{\beta}\right)^{4} h_{\alpha \beta(4)}+10\left(\mathcal{Q}-33 \mathcal{S}^{2}\right)\left(\mathcal{D}_{\alpha}{ }^{\beta}\right)^{2} h_{\alpha(3) \beta(2)}\right. \\
& \left.+\left(\mathcal{Q}-57 \mathcal{S}^{2}\right)\left(\mathcal{Q}-25 \mathcal{S}^{2}\right) h_{\alpha(5)}\right) \\
\mathfrak{C}_{\alpha(6)}(h)=\frac{1}{2^{5}}( & 6\left(\mathcal{D}_{\alpha}{ }^{\beta}\right)^{5} h_{\alpha \beta(5)}+20\left(\mathcal{Q}-48 \mathcal{S}^{2}\right)\left(\mathcal{D}_{\alpha}{ }^{\beta}\right)^{3} h_{\alpha(3) \beta(3)} \\
& \left.+\left(6 \mathcal{Q}^{2}-704 \mathcal{S}^{2}+18432 \mathcal{S}^{4} \mathcal{Q}\right) \mathcal{D}_{\alpha}{ }^{\beta} h_{\alpha(5) \beta}\right)
\end{aligned}
$$

\footnotetext{
${ }^{2}$ Relation (1.12) was obtained in [39] via the component reduction of the higher-spin $\mathcal{N}=1$ super-Cotton tensor, eq. (4.50), which is remarkably simple. This is an example of the power of supersymmetry.
} 
where $\mathcal{Q}$ is the second quadratic Casimir of the $\operatorname{AdS}$ group $\mathrm{SO}(2,2)$, given by eq. $(2.2 \mathrm{~b})$. No simple systematic method was offered in [7] to degauge $\mathfrak{C}_{\alpha(n)}(h)$ for generic $n$. In this paper we solve the problem of deriving a closed-form expression for $\mathfrak{C}_{\alpha(n)}(h)$ in terms of the AdS Lorentz covariant derivatives. An analogous result is also derived in the case of $\mathcal{N}=1 \mathrm{AdS}_{3}$ supersymmetry.

This paper is organised as follows. In section 2 we begin by reviewing the structure of on-shell partially massless and massive fields in $\mathrm{AdS}_{3}$. The Cotton tensors $\mathfrak{C}_{\alpha(n)}(h)$, for arbitrary $n \geq 2$, are then constructed and their factorisation into second (and first) order operators is discussed. In section 3 we use the results of section 2 to analyse the on-shell dynamics of (new) topologically massive higher-spin gauge models in $\mathrm{AdS}_{3}$. Section 4 is devoted to $\mathcal{N}=1$ supersymmetric extensions of the aforementioned results. Concluding remarks are given in section 5 . The main body is accompanied by three technical appendices. Appendix A lists our two-component spinor conventions. Appendix B describes the generating function formalism, which is the framework used to derive many of the results in this work. In appendix $\mathrm{C}$ we discuss some specific features of the higher-spin Cotton tensors in conformally flat backgrounds.

\section{Higher-spin Cotton tensors and on-shell fields in $\mathrm{AdS}_{3}$}

In this section we present a closed-form expression for the higher-spin Cotton tensor in $\mathrm{AdS}_{3}$ and give several applications of this result. Throughout this work, we use the twocomponent spinor formalism almost exclusively. The relevant conventions adopted in this paper are summarised in appendix A.

\section{$2.1 \quad \mathrm{AdS}_{3}$ geometry}

The covariant derivatives of $\mathrm{AdS}_{3}$ satisfy the commutation relations

$$
\left[\mathcal{D}_{a}, \mathcal{D}_{b}\right]=-4 \mathcal{S}^{2} M_{a b} \quad \Longleftrightarrow \quad\left[\mathcal{D}_{\alpha \beta}, \mathcal{D}_{\gamma \delta}\right]=4 \mathcal{S}^{2}\left(\varepsilon_{\gamma(\alpha} M_{\beta) \delta}+\varepsilon_{\delta(\alpha} M_{\beta) \gamma}\right)
$$

Here the parameter $\mathcal{S}$ is related to the AdS radius $\ell$ and the scalar curvature $R$ via $\ell^{-1}=2 \mathcal{S}$ and $R=-24 \mathcal{S}^{2}$, respectively. The Lorentz generators with vector $\left(M_{a b}=-M_{b a}\right)$ and spinor $\left(M_{\alpha \beta}=M_{\beta \alpha}\right)$ indices are defined to act on a vector $V_{c}$ and a spinor $\Psi_{\gamma}$ as in (A.8). ${ }^{3}$ We parametrise the curvature in terms of $\mathcal{S}$ in order for the notation to be consistent with that used in $\mathcal{N}=1$ supergravity (4.8) and AdS superspace (4.20).

Isometries of $\mathrm{AdS}_{3}$ are generated by those solutions of the conformal Killing equation (1.4), which obey the additional restriction $\sigma[\zeta]=0$. They are called the Killing vector fields on $\mathrm{AdS}_{3}$. Given a tensor field $\varphi$ on $\mathrm{AdS}_{3}$, its $\mathrm{AdS}$ transformation law is obtained from (1.5) by setting $\sigma[\zeta]=0$.

\footnotetext{
${ }^{3}$ We will also make use of the Lorentz generator with a vector index, $M_{a}$. The three types of generators $M_{a}, M_{a b}$ and $M_{\alpha \beta}$ are related to each other as follows: $-K^{a} M_{a}=\frac{1}{2} K^{a b} M_{a b}=\frac{1}{2} K^{\alpha \beta} M_{\alpha \beta}$. These relations follow the general rule (A.7).
} 
There are two quadratic Casimir operators of the $\mathrm{AdS}_{3}$ isometry algebra $\mathfrak{s o}(2,2) \cong$ $\mathfrak{s l}(2, \mathbb{R}) \oplus \mathfrak{s l}(2, \mathbb{R})$, and they can be chosen as follows (see, e.g., [50]):

$$
\begin{array}{ll}
\mathcal{F}:=\mathcal{D}^{\alpha \beta} M_{\alpha \beta}, & {\left[\mathcal{F}, \mathcal{D}_{\alpha(2)}\right]=0,} \\
\mathcal{Q}:=\square-2 \mathcal{S}^{2} M^{\alpha \beta} M_{\alpha \beta}, & {\left[\mathcal{Q}, \mathcal{D}_{\alpha(2)}\right]=0,}
\end{array}
$$

where $\square=\mathcal{D}^{a} \mathcal{D}_{a}=-\frac{1}{2} \mathcal{D}^{\alpha \beta} \mathcal{D}_{\alpha \beta}$. These Casimir operators are independent of one another, which may be seen by the action of $\mathcal{F}^{2}$ on an unconstrained completely symmetric rank- $n$ spinor field $h_{\alpha(n)}=h_{\left(\alpha_{1} \ldots \alpha_{n}\right)}$ :

$$
\mathcal{F}^{2} h_{\alpha(n)}=n^{2}\left[\mathcal{Q}-(n-2)(n+2) \mathcal{S}^{2}\right] h_{\alpha(n)}+n(n-1) \mathcal{D}_{\alpha(2)} \mathcal{D}^{\beta(2)} h_{\alpha(n-2) \beta(2)},
$$

for any integer $n \geq 0$. It should be pointed out that the divergence in the second term of (2.3) is not defined for $n=0$ and 1 , however the corresponding numerical coefficient is equal to zero in both cases.

\subsection{On-shell massive and partially massless fields}

We define an on-shell (real) field $h_{\alpha(n)}$, with $n \geq 2$, to be one which satisfies the first-order constraints $^{4}$

$$
\begin{aligned}
& 0=\mathcal{D}^{\beta \gamma} h_{\alpha(n-2) \beta \gamma}, \\
& 0=(\mathcal{F}-\rho) h_{\alpha(n)},
\end{aligned}
$$

for some mass parameter $\rho \in \mathbb{R}$. An equivalent form of the second irreducibility condition $(2.4 \mathrm{~b})$ is

$$
\mathcal{D}_{\left(\alpha_{1}\right.}{ }^{\beta} h_{\left.\alpha_{2} \ldots \alpha_{n}\right) \beta}=\frac{\rho}{n} h_{\alpha(n)} .
$$

The on-shell field $h_{\alpha(n)}$ is said to be transverse with pseudo-mass $\rho$, spin $n / 2$ and helicity $\sigma n / 2$, where $\sigma=\rho /|\rho|$. The equations (2.4a) and (2.5) were introduced in [51] (see also [50]). ${ }^{5}$

From the relation (2.3) we see that when restricted to the space of transverse fields, the quadratic Casimirs (2.2a) and (2.2b) are related via

$$
\mathcal{F}^{2} h_{\alpha(n)}=n^{2}\left(\mathcal{Q}-(n-2)(n+2) \mathcal{S}^{2}\right) h_{\alpha(n)} .
$$

Applying $\mathcal{F}$ to $(2.4 \mathrm{~b})$, we see that the on-shell conditions (2.4) lead to the following secondorder mass-shell equation

$$
0=\left(\mathcal{Q}-\left[(\rho / n)^{2}+(n-2)(n+2) \mathcal{S}^{2}\right]\right) h_{\alpha(n)} .
$$

In terms of the AdS d'Alembertian, $\square$, this reads

$$
0=\left(\square-\left[(\rho / n)^{2}-2(n+2) \mathcal{S}^{2}\right]\right) h_{\alpha(n)} .
$$

\footnotetext{
${ }^{4}$ For $n=0$ only (2.7) is present, whilst for $n=1$ there is only (2.4b).

${ }^{5}$ In the flat-space limit, these equations reduce to those considered in [52, 53].
} 
An on-shell field $h_{\alpha(n)}^{(t)}$ is said to be partially massless ${ }^{6}$ with depth $t$ if, in addition to the conditions (2.4), the pseudo-mass satisfies

$$
\rho \equiv \rho_{(t, n)}^{( \pm)}= \pm n(n-2 t) \mathcal{S}, \quad 1 \leq t \leq\lfloor n / 2\rfloor .
$$

We will refer to $\rho_{(t, n)}^{( \pm)}$as the depth- $t$ pseudo-mass values. ${ }^{7}$ The corresponding mass-shell equation (2.7) reduces to

$$
0=\left(\mathcal{Q}-\tau_{(t, n)} \mathcal{S}^{2}\right) h_{\alpha(n)}^{(t)}
$$

where the constants $\tau_{(t, n)}$ are the partially massless values defined by

$$
\tau_{(t, n)}=2 n(n-2 t)+4(t-1)(t+1) .
$$

The following identity holds for any transverse field $h_{\alpha(n)}$

$$
\left(\mathcal{F}-\rho_{(t, n)}^{(+)}\right)\left(\mathcal{F}-\rho_{(t, n)}^{(-)}\right) h_{\alpha(n)}=n^{2}\left(\mathcal{Q}-\tau_{(t, n)} \mathcal{S}^{2}\right) h_{\alpha(n)},
$$

and can be used to factorise second order operators involving the partially massless values into first order ones.

Using the formalism developed in appendix B, it may be shown that at the partially massless points (2.9), the system of equations (2.4) admits a depth- $t$ gauge symmetry of the form

$$
\delta_{\xi} h_{\alpha(n)}^{(t)}=\mathcal{D}_{\left(\alpha_{1} \alpha_{2}\right.} \cdots \mathcal{D}_{\alpha_{2 t-1} \alpha_{2 t}} \xi_{\left.\alpha_{2 t+1} \ldots \alpha_{n}\right)} .
$$

This is true only if the gauge parameter $\xi_{\alpha(n-2 t)}$ is also on-shell with the same pseudo-mass ${ }^{8}$

$$
\begin{aligned}
& 0=\mathcal{D}^{\beta \gamma} \xi_{\alpha(n-2 t-2) \beta \gamma}, \\
& 0=\left(\mathcal{F}-\rho_{(t, n)}^{( \pm)}\right) \xi_{\alpha(n-2 t)} .
\end{aligned}
$$

We note that strictly massless fields $h_{\alpha(n)}$ correspond to those partially massless fields with the minimal depth $t=1$ and therefore have pseudo-mass equal to $\rho_{(1, n)}^{( \pm)}$. They are defined modulo the standard first order gauge transformations,

$$
\delta_{\xi} h_{\alpha(n)}=\mathcal{D}_{\left(\alpha_{1} \alpha_{2}\right.} \xi_{\left.\alpha_{3} \ldots \alpha_{n}\right)} .
$$

In the interest of making contact with the representation theory of the $\mathrm{AdS}_{3}$ isometry group $\mathrm{SO}(2,2)$, it is useful to recast eq. (2.8) into the notation of [51],

$$
0=\left(\square+\ell^{-2}\left[n / 2+1-\eta^{-2}\right]\right) h_{\alpha(n)}, \quad \ell^{-1}:=2 \mathcal{S}, \quad \eta^{-1}:=\frac{\rho}{2 n \mathcal{S}} .
$$

\footnotetext{
${ }^{6}$ Partially massless fields have been studied in diverse dimensions for over 35 years, see [54-61] for some of the earlier works. Lagrangian models for partially massless fields in $\mathrm{AdS}_{d}$ were constructed in [62-64], and in $[65,66]$ for the specific case of $\mathrm{AdS}_{3}$.

${ }^{7}$ We note that the \pm in $(2.9)$ coincides the sign of the helicity, since $\sigma=\rho_{(t, n)}^{( \pm)} /\left|\rho_{(t, n)}^{( \pm)}\right|= \pm 1$. The positive and negative branches are related via the identity $\rho_{(\lfloor n / 2\rfloor+t, n)}^{(+)}=\rho_{(\lceil n / 2\rceil-t, n)}^{(-)}$for arbitrary $t$.

${ }^{8}$ The system of equations (2.14a) and (2.14b) is defined only if $n-2 t \geq 2$. For $n-2 t=1$ only eq. (2.14b) is present. If $n-2 t=0$, then these conditions must be replaced with $0=\left(\mathcal{Q}-(n-2)(n+2) \mathcal{S}^{2}\right) \xi$.
} 
In this context, the minimal energy of the corresponding $\mathfrak{s o}(2,2)$ irreducible representation $^{9}$ is $E_{0}=1+\left|\eta^{-1}\right|$, and the unitarity bound is $E_{0} \geq n / 2$, or equivalently $|\rho| \geq n(n-2) \mathcal{S}$. The minimal energy of the partially massless depth- $t$ field $h_{\alpha(n)}^{(t)}$ is $E_{0}^{(t, n)}=n / 2+(1-$ t). Therefore, strictly massless fields saturate the unitarity bound, whilst true partially massless fields violate it (they are non-unitary). Finally, we note that the physical mass (see e.g. [68]) is related to the pseudo-mass via

$$
\rho_{\text {phys }}^{2}=(\rho / n)^{2}-\left(\rho_{(1, n)}^{( \pm)} / n\right)^{2} .
$$

In terms of $\rho_{\text {phys }}$, the unitarity bound is $\rho_{\text {phys }}^{2} \geq 0$. Partially massless fields are thus seen to have negative physical mass-squared: $\left(\rho_{\text {phys }}^{(t, n)}\right)^{2}=-4(t-1)(n-t-1) \mathcal{S}^{2}$.

\subsection{Linearised higher-spin Cotton tensors}

It is a difficult technical problem to obtain an explicit expression for $\mathfrak{C}_{\alpha(n)}(h)$ in terms of the AdS covariant derivative. In particular, the minimal uplift of (1.12) is not gauge invariant or transverse. To restore these properties, (1.12) must be supplemented with curvature dependent terms, as in the low spin cases (1.14). However, for generic spin, previous attempts to do this were unsuccessful [7, 42]. In order to derive the Cotton tensors in $\mathrm{AdS}_{3}$, it is advantageous to recast the problem into the framework of homogeneous polynomials (or generating functions). The basics of this formalism are discussed in appendix B.

We begin by making an ansatz for the higher-spin Cotton tensor, which must be done separately for the bosonic $(n=2 s)$ and fermionic $(n=2 s+1)$ cases. In terms of homogeneous polynomials, our ansätze are as follows

$$
\begin{aligned}
\mathfrak{C}_{(2 s)}(h) & =\sum_{j=0}^{s-1} b_{j} \prod_{t=1}^{j}\left(\mathcal{Q}-\tau_{(s-t, 2 s)} \mathcal{S}^{2}\right) \mathcal{D}_{(2)}^{s-j-1} \mathcal{D}_{(0)} \mathcal{D}_{(-2)}^{s-j-1} h_{(2 s)}, \\
\mathfrak{C}_{(2 s+1)}(h) & =\sum_{j=0}^{s} f_{j} \prod_{t=1}^{j}\left(\mathcal{Q}-\tau_{(s-t+1,2 s+1)} \mathcal{S}^{2}\right) \mathcal{D}_{(2)}^{s-j} \mathcal{D}_{(-2)}^{s-j} h_{(2 s+1)},
\end{aligned}
$$

for undetermined real coefficients $b_{j}$ and $f_{j}$, and where $\tau_{(t, n)}$ are the partially massless values (2.11). We refer the reader to appendix B for an explanation of the notation. The main motivation for (2.18) is the expectation ${ }^{10}$ that in an AdS background, the gauge invariant action (2.23) for a conformal higher-spin field $h_{\alpha(n)}$ should factorise (in the transverse gauge) into products of minimal second-order operators involving all partial mass values. The factorisation of the Cotton tensors will be elaborated on in the next section.

To fix the coefficients in (2.18), we require that the expressions (2.18a) and (2.18b) are transverse (1.8) and gauge invariant (1.9). The former condition takes the form $0=$ $\mathcal{D}_{(-2)} \mathfrak{C}_{(n)}(h)$ which, upon employing the identity (B.5b), yields the recurrence relations

$$
\begin{aligned}
& 0=b_{j}-4(s-j)(s+j) b_{j-1}, \quad 1 \leq j \leq s-1, \\
& 0=f_{j}-4(s-j+1)(s+j) f_{j-1}, \quad 1 \leq j \leq s .
\end{aligned}
$$

\footnotetext{
${ }^{9}$ The unitary irreducible representations of $\mathfrak{s o}(2,2)$ are denoted $D\left(E_{0}, s\right)$, where $E_{0}$ is the minimal energy and $s$ the helicity, see [67] and references therein. The unitary bound is $E_{0} \geq|s|$.

${ }^{10}$ Strictly speaking, to the best of our knowledge, most results regarding the factorisation of the conformal higher-spin kinetic operators apply only in even dimensions and only in the bosonic case.
} 
These two systems determine the coefficients $b_{j}$ and $f_{j}$ up to an overall normalisation:

$$
\begin{aligned}
& b_{j}=2^{2 j}\left(\begin{array}{c}
s+j \\
2 j+1
\end{array}\right) \frac{(2 j+1) !}{s} b_{0}, \\
& f_{j}=2^{2 j}\left(\begin{array}{c}
s+j \\
2 j
\end{array}\right)(2 j) ! f_{0} .
\end{aligned}
$$

To ensure that $\mathfrak{C}_{\alpha(n)}(h)$ reduces to $(1.12)$ in the flat limit, we choose $b_{0}$ and $f_{0}$ as follows

$$
b_{0}=\frac{1}{2^{2 s-1}(2 s-1) !}, \quad f_{0}=\frac{1}{2^{2 s}(2 s) !} .
$$

It is interesting to note that even though the imposition of transversality completely fixes the coefficients in (2.18), the resulting descendent is automatically gauge invariant (and vice versa). This can be attributed to the symmetry property (2.24) and the Noether identity which follows as a consequence. Gauge invariance of (2.18), with the coefficients given by (2.20), can also be checked explicitly by expressing the gauge transformations in the form $\delta_{\xi} h_{(n)} \propto \mathcal{D}_{(2)} \xi_{(n-2)}$ and using the identity (B.5a).

To conclude this section, we provide the final expressions for the higher-spin Cotton tensors with explicit indices. They are given by

$$
\begin{aligned}
\mathfrak{C}_{\alpha(2 s)}(h)=\frac{1}{2^{2 s-1}} \sum_{j=0}^{s-1} 2^{2 j+1}\left(\begin{array}{c}
s+j \\
2 j+1
\end{array}\right) \prod_{t=1}^{j}\left(\mathcal{Q}-\tau_{(s-t, 2 s)} \mathcal{S}^{2}\right) \\
\times \mathcal{D}_{\alpha(2)}^{s-j-1} \mathcal{D}_{\alpha}^{\beta}\left(\mathcal{D}^{\beta(2)}\right)^{s-j-1} h_{\alpha(2 j+1) \beta(2 s-2 j-1)}, \\
\mathfrak{C}_{\alpha(2 s+1)}(h)=\frac{1}{2^{2 s}} \sum_{j=0}^{s} 2^{2 j}\left(\begin{array}{c}
s+j \\
2 j
\end{array}\right) \frac{(2 s+1)}{(2 j+1)} \prod_{t=1}^{j}\left(\mathcal{Q}-\tau_{(s-t+1,2 s+1)} \mathcal{S}^{2}\right) \\
\times \mathcal{D}_{\alpha(2)}^{s-j}\left(\mathcal{D}^{\beta(2)}\right)^{s-j} h_{\alpha(2 j+1) \beta(2 s-2 j)},
\end{aligned}
$$

in the bosonic and fermionic cases respectively.

\subsection{Factorisation of the Cotton tensors and CHS action}

The higher-spin Cotton tensors (2.22) are both gauge invariant and transverse. This means that the action for the conformal higher-spin field $h_{\alpha(n)}$,

$$
S_{\mathrm{CS}}^{(n)}[h]=\frac{\mathrm{i}^{n}}{2^{\lceil n / 2\rceil+1}} \int \mathrm{d}^{3} x e h^{\alpha(n)} \mathfrak{C}_{\alpha(n)}(h), \quad e^{-1}:=\operatorname{det}\left(e_{a}{ }^{m}\right),
$$

which is of the Chern-Simons type, is gauge invariant. Since $h_{\alpha(n)}$ and $\mathfrak{C}_{\alpha(n)}(h)$ are primary fields with dimension (1.6) and (1.10) respectively, the action is conformally invariant. Furthermore, (2.23) is symmetric in the sense that, modulo a total derivative, the relation

$$
\int \mathrm{d}^{3} x e g^{\alpha(n)} \mathfrak{C}_{\alpha(n)}(h)=\int \mathrm{d}^{3} x e h^{\alpha(n)} \mathfrak{C}_{\alpha(n)}(g)
$$

holds for arbitrary fields $g_{\alpha(n)}$ and $h_{\alpha(n)}$. 
Since the action is gauge invariant, we may impose the transverse gauge condition

$$
h_{\alpha(n)} \equiv h_{\alpha(n)}^{\mathrm{T}}, \quad 0=\mathcal{D}^{\beta(2)} h_{\beta(2) \alpha(n-2)}^{\mathrm{T}} .
$$

When the prepotential is transverse, only the $j=\lceil n / 2\rceil-1$ term contributes to (2.22), and the corresponding Cotton tensors reduce to

$$
\begin{aligned}
\mathfrak{C}_{\alpha(2 s)}\left(h^{\mathrm{T}}\right) & =\prod_{t=1}^{s-1}\left(\mathcal{Q}-\tau_{(t, 2 s)} \mathcal{S}^{2}\right) \mathcal{D}_{\alpha}{ }^{\beta} h_{\alpha(2 s-1) \beta}^{\mathrm{T}}, \\
\mathfrak{C}_{\alpha(2 s+1)}\left(h^{\mathrm{T}}\right) & =\prod_{t=1}^{s}\left(\mathcal{Q}-\tau_{(t, 2 s+1)} \mathcal{S}^{2}\right) h_{\alpha(2 s+1)}^{\mathrm{T}} .
\end{aligned}
$$

As a result, in this gauge the fermionic conformal higher-spin action (2.23) fully factorises into products of minimal second order operators involving partial mass values of all possible depths. From (2.26a) it is clear that the bosonic Cotton tensor does not wholly factorise and does not include the maximal depth partial mass $\tau_{(s, 2 s)}$ as a factor. However, in this case one may show that the following descendent of $\mathfrak{C}_{\alpha(2 s)}(h)$ fully factorises

$$
\mathcal{D}_{\alpha}{ }^{\beta} \mathfrak{C}_{\alpha(2 s-1) \beta}\left(h^{\mathrm{T}}\right)=\prod_{t=1}^{s}\left(\mathcal{Q}-\tau_{(t, 2 s)} \mathcal{S}^{2}\right) h_{\alpha(2 s)}^{\mathrm{T}},
$$

and its factors include all possible partial mass values.

Using the identity (2.12), from (2.26) one may derive the following useful alternative forms for the Cotton tensors,

$$
\begin{aligned}
\mathfrak{C}_{\alpha(2 s)}\left(h^{\mathrm{T}}\right) & =\frac{1}{(2 s)^{2 s-1}} \mathcal{F} \prod_{t=1}^{s-1}\left(\mathcal{F}-\rho_{(t, 2 s)}^{(-)}\right)\left(\mathcal{F}-\rho_{(t, 2 s)}^{(+)}\right) h_{\alpha(2 s)}^{\mathrm{T}}, \\
\mathfrak{C}_{\alpha(2 s+1)}\left(h^{\mathrm{T}}\right) & =\frac{1}{(2 s+1)^{2 s}} \prod_{t=1}^{s}\left(\mathcal{F}-\rho_{(t, 2 s+1)}^{(-)}\right)\left(\mathcal{F}-\rho_{(t, 2 s+1)}^{(+)}\right) h_{\alpha(2 s+1)}^{\mathrm{T}} .
\end{aligned}
$$

We see that $\mathfrak{C}_{\alpha(n)}(h)$ factorises into products of first-order differential operators involving all partial pseudo-mass values. ${ }^{11}$

\subsection{Partially massless prepotentials}

From the above expressions for $\mathfrak{C}_{\alpha(n)}(h)$, it is clear that the Cotton tensor of any on-shell partially massless field vanishes. More specifically, if $h_{\alpha(n)} \equiv h_{\alpha(n)}^{(t)}$ satisfies the on-shell conditions (2.4) and has depth- $t$, i.e. $\rho$ is given by (2.9), then

$$
\mathfrak{C}_{\alpha(n)}(h)=0 .
$$

In Minkowski space, it is known (see [36, 43] and appendix C) that the vanishing of the Cotton tensor associated with the field $h_{\alpha(n)}$, is a necessary and sufficient condition for

\footnotetext{
${ }^{11}$ We note that $\rho_{(s, 2 s)}^{( \pm)}=0$, so that the maximal-depth bosonic pseudo-mass also appears in (2.28a).
} 
$h_{\alpha(n)}$ to be pure gauge. As argued in appendix C, an analogous statement holds in AdS space:

$$
\mathfrak{C}_{\alpha(n)}(h)=0 \quad \Longleftrightarrow \quad h_{\alpha(n)}=\mathcal{D}_{\alpha(2)} \xi_{\alpha(n-2)},
$$

for some $\xi_{\alpha(n-2)}$. This provides another way to understand why partially massless fields in $\mathrm{AdS}_{3}$ do not have any local dynamical degrees of freedom.

In fact, for on-shell partially massless fields $h_{\alpha(n)}^{(t)}$, the statement (2.30) can be taken a step further: writing $h_{\alpha(n)}^{(t)}=\mathcal{D}_{\alpha(2)} \xi_{\alpha(n-2)}$ and requiring this to be transverse yields the equation

$$
0=\left(\tau_{(t, n)}-\tau_{(1, n)}\right) \mathcal{S}^{2} \xi_{\alpha(n-2)}-\frac{(n-2)(n-3)}{4(n-1)} \mathcal{D}_{\alpha(2)} \mathcal{D}^{\beta(2)} \xi_{\alpha(n-4) \beta(2)} .
$$

Here we have made use of the identities (B.5a) and (2.10). Therefore, if $t=1$ (i.e. $h$ is massless) then $\xi_{\alpha(n-2)}$ is transverse. On the other hand, if $2 \leq t \leq\lfloor n / 2\rfloor$ then (2.31) implies

$$
\xi_{\alpha(n-2)}=\mathcal{D}_{\alpha(2)} \xi_{\alpha(n-4)} \quad \Longrightarrow \quad h_{\alpha(n)}^{(t)}=\mathcal{D}_{\alpha(2)} \mathcal{D}_{\alpha(2)} \xi_{\alpha(n-4)}
$$

for some non-zero $\xi_{\alpha(n-4)}$. Once again, requiring $h_{\alpha(n)}^{(t)}$ to be transverse yields the equation

$$
0=\left(\tau_{(t, n)}-\tau_{(2, n)}\right) \mathcal{S}^{2} \mathcal{D}_{\alpha(2)} \xi_{\alpha(n-4)}-\frac{(n-4)(n-5)}{8(n-2)} \mathcal{D}_{\alpha(2)} \mathcal{D}_{\alpha(2)} \mathcal{D}^{\beta(2)} \xi_{\alpha(n-6) \beta(2)}
$$

and similar remarks hold. This procedure terminates when $t$ gradients have been extracted, whereupon $h_{\alpha(n)}^{(t)}$ takes the form

$$
h_{\alpha(n)}^{(t)}=\underbrace{\mathcal{D}_{\alpha(2)} \cdots \mathcal{D}_{\alpha(2)}}_{t \text {-times }} \xi_{\alpha(n-2 t)},
$$

for some non-zero $\xi_{\alpha(n-2 t)}$ being also on-shell (i.e. satisfying (2.14)). The partially massless field $h_{\alpha(n)}^{(t)}$ will be said to be 'pure depth- $t$ gauge'.

\section{Massive higher-spin gauge models}

In this section we demonstrate that the properties of the higher-spin Cotton tensors allow us to analyse the on-shell dynamics of massive higher-spin gauge-invariant actions in $\mathrm{AdS}_{3}$.

\subsection{New topologically massive higher-spin gauge models}

The so-called 'new topologically massive' (NTM) models for bosonic fields were first introduced in [33] in Minkowski space. Extensions of these models to fields with half-integer spin were proposed in [42], where their generalisations to an AdS background were also given. These models are formulated solely in terms of the gauge prepotentials $h_{\alpha(n)}$ and the associated Cotton tensors $\mathfrak{C}_{\alpha(n)}(h)$. Having obtained explicit expressions for the latter in AdS, we now take a closer look at these models. 
Given an integer $n \geq 2$, the gauge-invariant NTM action for the field $h_{\alpha(n)}$ given in $[7]$ is

$$
S_{\mathrm{NTM}}^{(n)}[h]=\frac{\mathrm{i}^{n}}{2^{\lceil n / 2\rceil+1}} \frac{1}{\rho} \int \mathrm{d}^{3} x e \mathfrak{C}^{\alpha(n)}(h)(\mathcal{F}-\rho) h_{\alpha(n)},
$$

where $\rho$ is some arbitrary mass parameter. The equation of motion obtained by varying (3.1) with respect to the field $h_{\alpha(n)}$ is

$$
0=(\mathcal{F}-\rho) \mathfrak{C}_{\alpha(n)}(h) .
$$

We may analyse the solutions to (3.2) in terms of (i) the gauge prepotential $h_{\alpha(n)}$; or (ii) its field strength $\mathfrak{C}_{\alpha(n)}(h)$.

Let us first work in terms of the prepotential. Since the action (3.1) is invariant under the gauge transformations (1.7), we may impose the transverse gauge condition (2.25), whereupon the field equation (3.2) takes the form

$$
\begin{aligned}
& 0=(\mathcal{F}-\rho) \mathcal{F} \prod_{t=1}^{s-1}\left(\mathcal{F}-\rho_{(t, 2 s)}^{(-)}\right)\left(\mathcal{F}-\rho_{(t, 2 s)}^{(+)}\right) h_{\alpha(2 s)}^{\mathrm{T}}, \\
& 0=(\mathcal{F}-\rho) \prod_{t=1}^{s}\left(\mathcal{F}-\rho_{(t, 2 s+1)}^{(-)}\right)\left(\mathcal{F}-\rho_{(t, 2 s+1)}^{(+)}\right) h_{\alpha(2 s+1)}^{\mathrm{T}},
\end{aligned}
$$

in the bosonic and fermionic cases respectively. For simplicity, here we will only seek particular solutions to (3.3), rather than general ones. Clearly, any $h_{\alpha(n)}^{\mathrm{T}}$ satisfying

$$
0=(\mathcal{F}-\rho) h_{\alpha(n)}^{\mathrm{T}}, \quad \rho \neq \rho_{(t, n)}^{( \pm)},
$$

is a solution to (3.3). In addition, any $h_{\alpha(n)}^{\mathrm{T}} \equiv h_{\alpha(n)}^{(t)}$ satisfying

$$
0=\left(\mathcal{F}-\rho_{(t, n)}^{( \pm)}\right) h_{\alpha(n)}^{(t)},
$$

for some $1 \leq t \leq\lfloor n / 2\rfloor$, is also a solution. However, in this case its Cotton tensor vanishes

$$
0=\mathfrak{C}_{\alpha(n)}\left(h^{(t)}\right)
$$

As discussed section 2.4, this condition is necessary and sufficient to conclude that $h_{\alpha(n)}^{(t)}$ is pure depth- $t$ gauge. Hence, of these solutions, the only non-trivial one is (3.4), which propagates a single degree of freedom with pseudo-mass $\rho$, spin $n / 2$ and helicity $\operatorname{sgn}(\rho) n / 2$.

If instead we wish to analyse solutions to (3.2) in terms of the field strength $\mathfrak{C}_{\alpha(n)}(h)$, then the analysis should be split into two cases. First, if $\mathfrak{C}_{\alpha(n)}(h)$ satisfies

$$
0=(\mathcal{F}-\rho) \mathfrak{C}_{\alpha(n)}(h), \quad \rho \neq \rho_{(t, n)}^{( \pm)},
$$

then, in accordance with section 2.2, eq. (3.7) together with the conservation identity (1.8) means that the field strength $\mathfrak{C}_{\alpha(n)}(h)$ itself defines an on-shell spin $n / 2$ field with pseudomass $\rho$, and helicity $\operatorname{sgn}(\rho) n / 2$. Next we consider the case when $\mathfrak{C}_{\alpha(n)}(h)$ satisfies

$$
0=\left(\mathcal{F}-\rho_{(t, n)}^{( \pm)}\right) \mathfrak{C}_{\alpha(n)}(h),
$$


for some $1 \leq t \leq\lfloor n / 2\rfloor$. Now let us examine the Cotton tensor of the field strength $\mathfrak{C}_{\alpha(n)}(h)$, which for ease of notation we denote $\mathfrak{G}_{\alpha(n)}(h)$. Since $\mathfrak{C}_{\alpha(n)}(h)$ is identically conserved, eq. (1.8), its Cotton tensor factorises according to (2.28),

$$
\begin{aligned}
\mathfrak{G}_{\alpha(2 s)}(h) & =\frac{1}{(2 s)^{2 s-1}} \mathcal{F} \prod_{t=1}^{s-1}\left(\mathcal{F}-\rho_{(t, 2 s)}^{(-)}\right)\left(\mathcal{F}-\rho_{(t, 2 s)}^{(+)}\right) \mathfrak{C}_{\alpha(2 s)}(h), \\
\mathfrak{G}_{\alpha(2 s+1)}(h) & =\frac{1}{(2 s+1)^{2 s}} \prod_{t=1}^{s}\left(\mathcal{F}-\rho_{(t, 2 s+1)}^{(-)}\right)\left(\mathcal{F}-\rho_{(t, 2 s+1)}^{(+)}\right) \mathfrak{C}_{\alpha(2 s+1)}(h) .
\end{aligned}
$$

On account of $(3.8)$, we conclude that $\mathfrak{G}_{\alpha(n)}(h)=0$. Therefore, $\mathfrak{C}_{\alpha(n)}(h)$ is itself pure gauge:

$$
\mathfrak{C}_{\alpha(n)}(h)=\mathcal{D}_{\alpha(2)} \widehat{\mathfrak{C}}_{\alpha(n-2)}(h),
$$

for some $\widehat{\mathfrak{C}}_{\alpha(n-2)}(h)$. Since $\mathfrak{C}_{\alpha(n)}(h)$ is gauge invariant, we can take $h_{\alpha(n)}$ in (3.10) to be transverse, whereupon the left hand side of (3.10) factorises. Consequently, the only way that $\mathfrak{C}_{\alpha(n)}(h)$ can have the form (3.10), is if $h_{\alpha(n)}$ is pure gauge, and hence $\mathfrak{C}_{\alpha(n)}(h)=0$.

Therefore, from both points of view, the conclusion is that if the pseudo-mass $\rho$ in (3.1) takes on one of the partially massless values, then it describes only pure gauge degrees of freedom. If however $\rho$ satisfies $\rho \neq \rho_{(t, n)}^{( \pm)}$, but is otherwise arbitrary, ${ }^{12}$ then on-shell the model describes a spin $n / 2$ mode with pseudo-mass $\rho$ and helicity $\operatorname{sgn}(\rho) n / 2$.

\subsection{Topologically massive higher-spin gauge models}

Topologically massive models for higher-spin gauge fields are higher-spin extensions of linearised topologically massive gravity [69, 70], and were proposed in [42] in both Minkowski and AdS space. They are obtained by coupling the $\mathrm{AdS}_{3}$ counterparts of the massless Fronsdal [71] and Fang-Fronsdal [72] actions to the bosonic (even $n$ ) and fermionic (odd $n$ ) Chern-Simons action (2.23), respectively. However, since the latter is constructed using the higher-spin Cotton tensor, whose explicit form was not known at the time of writing [42], the on-shell analysis was restricted to the case of Minkowski space only. In this section we address this issue and take a brief look at the on-shell content of these models in $\mathrm{AdS}_{3}$.

There are two types of the higher-spin massless actions, first-order and second-order ones. Given an integer $s \geq 2$, the first-order model is described by real fields $h_{\alpha(2 s+1)}$, $y_{\alpha(2 s-1)}$ and $z_{\alpha(2 s-3)}$, which are defined modulo gauge transformations of the form

$$
\begin{aligned}
\delta_{\xi} h_{\alpha(2 s+1)} & =\mathcal{D}_{\alpha(2)} \xi_{\alpha(2 s-1)}, \\
\delta_{\xi} y_{\alpha(2 s-1)} & =\frac{1}{(2 s+1)(2 s-1)}\left(\mathcal{F}-\rho_{(1,2 s+1)}^{(-)}\right) \xi_{\alpha(2 s-1)}, \\
\delta_{\xi} z_{\alpha(2 s-3)} & =\mathcal{D}^{\beta(2)} \xi_{\alpha(2 s-3) \beta(2)} .
\end{aligned}
$$

\footnotetext{
${ }^{12}$ For unitarity one should restrict $\rho$ to satisfy $|\rho|>n(n-2) \mathcal{S}$.
} 
The three-dimensional counterpart of the Fang-Fronsdal action is ${ }^{13}$

$$
\begin{aligned}
& S_{\mathrm{FF}}^{(2 s+1)}[h, y, z]=\left(-\frac{1}{2}\right)^{s} \frac{\mathrm{i}}{2} \int \mathrm{d}^{3} x e\left\{\frac{1}{(2 s+1)} h^{\alpha(2 s+1)}\left(\mathcal{F}-\rho_{(1,2 s+1)}^{(-)}\right) h_{\alpha(2 s+1)}\right. \\
& +4 y^{\alpha(2 s-1)}\left(\mathcal{F}-\rho_{(1,2 s+1)}^{(+)}\right) y_{\alpha(2 s-1)}-\frac{(s-1)}{s(2 s-1)} z^{\alpha(2 s-3)}\left(\mathcal{F}-\rho_{(1,2 s+1)}^{(-)}\right) z_{\alpha(2 s-3)} \\
& \left.+2 y^{\alpha(2 s-1)}\left[(2 s-1) \mathcal{D}^{\beta(2)} h_{\alpha(2 s-1) \beta(2)}-\frac{(2 s+1)(s-1)}{s} \mathcal{D}_{\alpha(2)} z_{\alpha(2 s-3)}\right]\right\} .
\end{aligned}
$$

This model can be reformulated in terms of a single reducible tensor field $\boldsymbol{h}_{\beta(2), \alpha(n-2)}$, which corresponds to the AdS extension of the massless model introduced by Tyutin and Vasiliev [53].

Given an integer $s \geq 2$, the second-order model is described by the real fields $h_{\alpha(2 s)}$ and $y_{\alpha(2 s-4)}$, which are defined modulo gauge transformations of the form

$$
\begin{aligned}
\delta_{\xi} h_{\alpha(2 s)} & =\mathcal{D}_{\alpha(2)} \xi_{\alpha(2 s-2)}, \\
\delta_{\xi} y_{\alpha(2 s-4)} & =\frac{2 s-2}{2 s-1} \mathcal{D}^{\beta(2)} \xi_{\alpha(2 s-4) \beta(2)} .
\end{aligned}
$$

The corresponding gauge-invariant Fronsdal-type action is

$$
\begin{aligned}
& S_{\mathrm{F}}^{(2 s)}[h, y]=\frac{1}{2}\left(-\frac{1}{2}\right)^{s} \int \mathrm{d}^{3} x e\left\{h^{\alpha(2 s)}\left(\mathcal{Q}-\tau_{(1,2 s)} \mathcal{S}^{2}\right) h_{\alpha(2 s)}+\frac{s}{2} h^{\alpha(2 s)} \mathcal{D}_{\alpha(2)} \mathcal{D}^{\beta(2)} h_{\alpha(2 s-2) \beta(2)}\right. \\
& -\frac{(2 s-3)}{2} y^{\alpha(2 s-4)} \mathcal{D}^{\beta(2)} \mathcal{D}^{\beta(2)} h_{\alpha(2 s-4) \beta(4)}-\frac{(2 s-3)}{s}\left[y^{\alpha(2 s-4)}\left(\mathcal{Q}-\tau_{(2,2 s)} \mathcal{S}^{2}\right) y_{\alpha(2 s-4)}\right. \\
& \left.\left.-\frac{(s-2)(2 s-5)}{8(s-1)} y^{\alpha(2 s-4)} \mathcal{D}_{\alpha(2)} \mathcal{D}^{\beta(2)} y_{\alpha(2 s-6) \beta(2)}\right]\right\}
\end{aligned}
$$

For spin $s+1 / 2$ and $s$ respectively, the corresponding topologically massive models are described by the gauge-invariant actions

$$
\begin{aligned}
S_{\mathrm{TM}}^{(2 s+1)}[h, y, z] & =S_{\mathrm{CS}}^{(2 s+1)}[h]-\mu(\rho, s) S_{\mathrm{FF}}^{(2 s+1)}[h, y, z], \\
S_{\mathrm{TM}}^{(2 s)}[h, y] & =S_{\mathrm{CS}}^{(2 s)}[h]-\nu(\rho, s) S_{\mathrm{F}}^{(2 s)}[h, y] .
\end{aligned}
$$

The functions $\mu(\rho, s)$ and $\nu(\rho, s)$ are defined by

$$
\begin{aligned}
& \mu(\rho, s)=\frac{1}{(2 s+1)^{2 s-1}}\left(\rho-\rho_{(1,2 s+1)}^{(+)}\right) \prod_{t=2}^{s}\left[\rho^{2}-\left(\rho_{(t, 2 s+1)}^{( \pm)}\right)^{2}\right], \\
& \nu(\rho, s)=\frac{1}{(2 s)^{2 s-3}}\left(\rho-\rho_{(s, 2 s)}^{( \pm)}\right) \prod_{t=2}^{s-1}\left[\rho^{2}-\left(\rho_{(t, 2 s)}^{( \pm)}\right)^{2}\right]
\end{aligned}
$$

where $\rho \in \mathbb{R}$ is some arbitrary real constant with mass dimension one. For unitarity, the mass parameter $\rho$ must satisfy $|\rho|>n(n-2) \mathcal{S}$. For such values the functions (3.16a)

\footnotetext{
${ }^{13}$ There are actually two gauge invariant first-order Fang-Fronsdal actions in $\mathrm{AdS}_{3}$ [73]. The other is obtained by interchanging $\rho_{(1,2 s+1)}^{(+)}$with $\rho_{(1,2 s+1)}^{(-)}$everywhere in (3.11) and (3.12), or equivalently, sending $\mathcal{S} \rightarrow-\mathcal{S}$.
} 
and (3.16b) are always non-vanishing. For the (non-unitary) true partially massless values $\rho=\rho_{(t, n)}^{( \pm)}$, with $2 \leq t \leq\lfloor n / 2\rfloor$, the coupling constants (3.16) vanish.

It may be shown that on-shell, the model $S_{\mathrm{TM}}^{(n)}$ describes an irreducible spin- $n / 2$ field with pseudo-mass $\rho$ and helicity whose sign is equal to $\rho /|\rho|$. Let us sketch how this can be seen in the bosonic case with $n=2 s$.

In analogy with the analysis of [42], using the equation of motion obtained by varying $(3.15 \mathrm{~b})$ with respect to $y_{\alpha(2 s-4)}$, it follows that we may impose the gauge

$$
y_{\alpha(2 s-4)}=0, \quad \mathcal{D}^{\beta(2)} h_{\beta(2) \alpha(2 s-2)}=0 .
$$

In this gauge the Cotton tensor factorises in accordance with section 2.4, and the equation of motion obtained by varying $(3.15 \mathrm{~b})$ with respect to $h_{\alpha(2 s)}$ is

$$
0=\left(\mathcal{F} \prod_{t=2}^{s-1}\left(\mathcal{Q}-\tau_{(t, 2 s)} \mathcal{S}^{2}\right)+2 s \nu(\rho, s)\right)\left(\mathcal{Q}-\tau_{(1,2 s)} \mathcal{S}^{2}\right) h_{\alpha(2 s)}
$$

There are two types of solutions to (3.18). The first type solves $0=\left(\mathcal{Q}-\tau_{(1,2 s)} \mathcal{S}^{2}\right) h_{\alpha(2 s)}$, in which case $h_{\alpha(2 s)}$ is massless with only pure gauge degrees of freedom. The second type of solutions are those satisfying

$$
0=\left(\mathcal{F} \prod_{t=2}^{s-1}\left(\mathcal{Q}-\tau_{(t, 2 s)} \mathcal{S}^{2}\right)+2 s \nu(\rho, s)\right) h_{\alpha(2 s)} .
$$

Once again, here we will only seek particular solutions to (3.19). For $\nu(\rho, s)$ defined as in $(3.16 \mathrm{~b})$, one can show that any $h_{\alpha(2 s)}$ satisfying

$$
0=(\mathcal{F}-\rho) h_{\alpha(2 s)} \quad \Longrightarrow \quad 0=\left(\mathcal{Q}-\left[\rho^{2} /(2 s)^{2}+4(s-1)(s+1) \mathcal{S}^{2}\right]\right) h_{\alpha(2 s)}
$$

where $\rho$ satisfies $\rho \neq \rho_{(t, 2 s)}^{( \pm)}$, but is otherwise arbitrary, solves (3.19). We note that the residual gauge symmetry preserving the gauge fixing condition (3.17) is described by (3.13a), where the gauge parameter satisfies

$$
0=\mathcal{D}^{\beta(2)} \xi_{\beta(2) \alpha(2 s-4)}, \quad 0=\left(\mathcal{F}-\rho_{(1,2 s)}^{( \pm)}\right) \xi_{\alpha(2 s-2)}
$$

For the massive solutions (3.20), the residual gauge symmetry is exhausted since both (3.20) and (3.21) imply $\xi_{\alpha(2 s-2)}=0$ for $\rho \neq \rho_{(t, 2 s)}^{( \pm)}$.

In accordance with section 2.2, the field $h_{\alpha(2 s)}$ satisfying the second equation in (3.17) and the first equation in (3.20) defines an on-shell spin- $s$ field with pseudo-mass $\rho$ and helicity $s \rho /|\rho|$. It is important to note that the above discussion assumes that the pseudomass does not take on any of the true partially massless values. If this is the case then the coupling constant $\nu$ vanishes, and we are left with the Chern-Simons sector, which describes only pure gauge degrees of freedom. The analysis in the fermionic case proceeds in a similar fashion and we do not repeat it here. 


\section{Supersymmetric higher-spin gauge models in $\mathrm{AdS}_{3}$}

In this section we present a closed-form expression for the linearised higher-spin superCotton tensor in $\mathcal{N}=1$ AdS superspace, $\operatorname{AdS}^{3 \mid 2}$, and give several applications of this result. Before turning to the main construction, some general comments are in order about the super-Cotton tensor in supergravity.

\subsection{Conformal supergravity and super-Cotton tensors}

When formulating three-dimensional $\mathcal{N}$-extended conformal supergravity in conformal superspace [5], all information about the corresponding superspace geometry is encoded in a single curvature superfield, which is the super-Cotton tensor $\mathcal{W}$. The functional structure of the super-Cotton tensor is $\mathcal{N}$-dependent. The $\mathcal{N}=1$ super-Cotton tensor [74] is a primary symmetric rank-3 spinor superfield $\mathcal{W}_{\alpha \beta \gamma}$ of dimension $5 / 2$, which obeys the conformally invariant constraint [5]

$$
\nabla^{\alpha} \mathcal{W}_{\alpha \beta \gamma}=0
$$

In the $\mathcal{N}=2$ case, the super-Cotton tensor [75, 76] is a primary symmetric rank-2 spinor superfield $\mathcal{W}_{\alpha \beta}$ of dimension 2, which obeys the Bianchi identity [5]

$$
\nabla^{\alpha I} \mathcal{W}_{\alpha \beta}=0
$$

In the $\mathcal{N}=3$ case, the super-Cotton tensor is a primary spinor superfield $\mathcal{W}_{\alpha}$ of dimension $3 / 2$ constrained by [5]

$$
\nabla^{\alpha I} \mathcal{W}_{\alpha}=0
$$

In the $\mathcal{N}=4$ case, the super-Cotton tensor is a primary scalar superfield $\mathcal{W}$ of dimension 1 constrained by [5]

$$
\nabla^{\alpha I} \nabla_{\alpha}^{J} \mathcal{W}=\frac{1}{4} \delta^{I J} \nabla^{\alpha K} \nabla_{\alpha}^{K} \mathcal{W}
$$

For $\mathcal{N}>4$, the super-Cotton tensor [77, 78] is a completely antisymmetric tensor $\mathcal{W}^{I J K L}$ of dimension 1 constrained by [5]

$$
\nabla_{\alpha}^{I} \mathcal{W}^{J K L P}=\nabla_{\alpha}^{[I} \mathcal{W}^{J K L P]}-\frac{4}{\mathcal{N}-3} \nabla_{\alpha}^{Q} \mathcal{W}^{Q[J K L} \delta^{P] I}
$$

In the above relations, $\nabla_{\alpha}^{I}$ denotes the spinor covariant derivative of $\mathcal{N}$-extended conformal superspace [5].

The super-Cotton tensor is naturally encoded in the action for conformal supergravity, ${ }^{14} S_{\mathrm{CSG}}$, in the following sense [5, 79]:

$$
\mathcal{W} \propto \frac{\delta S_{\mathrm{CSG}}}{\delta H} .
$$

\footnotetext{
${ }^{14}$ For the $1 \leq \mathcal{N} \leq 6$ cases, the complete nonlinear actions for $\mathcal{N}$-extended conformal supergravity were derived in $[79,80]$ using the off-shell formulation for $\mathcal{N}$-extended conformal supergravity developed in [5]. The $\mathcal{N}=1$ and $\mathcal{N}=2$ conformal supergravity theories were constructed for the first time by van Nieuwenhuizen [81], and by Roček and van Nieuwenhuizen [82], respectively. The off-shell action for $\mathcal{N}=6$ conformal supergravity was independently derived by Nishimura and Tanii [83]. On-shell formulations for $\mathcal{N}$-extended conformal supergravity with $\mathcal{N}>2$ were given in [84, 85].
} 
Here $H$ denotes an unconstrained gauge prepotential for conformal supergravity. Modulo purely gauge degrees of freedom, the structure of unconstrained conformal gauge prepotentials are as follows: $H_{\alpha \beta \gamma}$ for $\mathcal{N}=1[86], H_{\alpha \beta}$ for $\mathcal{N}=2[75,76], H_{\alpha}$ for $\mathcal{N}=3$ [5], and $H$ for $\mathcal{N}=4[5]$.

In the case of $\mathcal{N}$-extended Poincare supersymmetry, higher-spin generalisations of the linearised super-Cotton tensor were given in [39, 40] for $\mathcal{N}=1$, in [38] for $\mathcal{N}=2$ and in [44] for $\mathcal{N}>2$. Below we generalise the results of $[39,40]$ to the $\mathcal{N}=1$ AdS supersymmetry, while the $\mathcal{N}>1$ case will be discussed elsewhere.

\subsection{Higher-spin super-Cotton tensors in conformally flat superspace}

In $\mathcal{N}=1$ supergravity, the covariant derivatives ${ }^{15}$

$$
\mathcal{D}_{A}=\left(\mathcal{D}_{a}, \mathcal{D}_{\alpha}\right)=E_{A}+\Omega_{A}=E_{A}{ }^{M} \partial_{M}+\frac{1}{2} \Omega_{A}{ }^{b c} M_{b c},
$$

obey the following graded commutation relations $[77,86]$

$$
\begin{aligned}
\left\{\mathcal{D}_{\alpha}, \mathcal{D}_{\beta}\right\}= & 2 \mathrm{i} \mathcal{D}_{\alpha \beta}-4 \mathrm{i} \mathcal{S} M_{\alpha \beta}, \\
{\left[\mathcal{D}_{\alpha \beta}, \mathcal{D}_{\gamma}\right]=} & -2 \varepsilon_{\gamma(\alpha} \mathcal{S} \mathcal{D}_{\beta)}+2 \varepsilon_{\gamma(\alpha} \mathcal{C}_{\beta) \delta \rho} M^{\delta \rho} \\
& +\frac{2}{3}\left(\mathcal{D}_{\gamma} \mathcal{S} M_{\alpha \beta}-4 \mathcal{D}_{(\alpha} \mathcal{S} M_{\beta) \gamma}\right),
\end{aligned}
$$

Here the torsion superfields $\mathcal{S}$ and $\mathcal{C}_{\alpha \beta \gamma}=\mathcal{C}_{(\alpha \beta \gamma)}$ are real and related to each other by the Bianchi identity $\mathcal{D}^{\gamma} \mathcal{C}_{\alpha \beta \gamma}=-\frac{4 \mathrm{i}}{3} \mathcal{D}_{\alpha \beta} \mathcal{S}$.

The algebra of covariant derivatives (4.8) is invariant under the following super-Weyl transformation [75, 87, 88]

$$
\begin{aligned}
& \delta_{\Sigma} \mathcal{D}_{\alpha}=\frac{1}{2} \Sigma \mathcal{D}_{\alpha}+\mathcal{D}^{\beta} \Sigma M_{\alpha \beta}, \\
& \delta_{\Sigma} \mathcal{D}_{a}=\Sigma \mathcal{D}_{a}+\frac{\mathrm{i}}{2}\left(\gamma_{a}\right)^{\gamma \delta} \mathcal{D}_{\gamma} \Sigma \mathcal{D}_{\delta}+\varepsilon_{a b c} \mathcal{D}^{b} \Sigma M^{c},
\end{aligned}
$$

where the parameter $\Sigma$ is a real unconstrained superfield. The corresponding super-Weyl transformations of the torsion tensors are

$$
\delta_{\Sigma} \mathcal{S}=\Sigma \mathcal{S}-\frac{\mathrm{i}}{4} \mathcal{D}^{2} \Sigma, \quad \delta_{\Sigma} \mathcal{C}_{\alpha \beta \gamma}=\frac{3}{2} \Sigma \mathcal{C}_{\alpha \beta \gamma}-\frac{\mathrm{i}}{2} \mathcal{D}_{(\alpha \beta} \mathcal{D}_{\gamma)} \Sigma
$$

The $\mathcal{N}=1$ super-Cotton tensor [74] is given by the expression

$$
\mathcal{W}_{\alpha \beta \gamma}=\left(\frac{\mathrm{i}}{2} \mathcal{D}^{2}+4 \mathcal{S}\right) \mathcal{C}_{\alpha \beta \gamma}+\mathrm{i} \mathcal{D}_{(\alpha \beta} \mathcal{D}_{\gamma)} \mathcal{S}
$$

Its super-Weyl transformation is $\delta_{\Sigma} \mathcal{W}_{\alpha \beta \gamma}=\frac{5}{2} \Sigma \mathcal{W}_{\alpha \beta \gamma}$, which means that $\mathcal{W}_{\alpha \beta \gamma}$ is a primary superfield.

\footnotetext{
${ }^{15}$ In the hope that no confusion arises, we use the same notation for the vector derivative (1.2) and its $\mathcal{N}=1$ analogue.
} 
Let $\mathcal{M}^{3 \mid 2}$ be a conformally flat superspace,

$$
\mathcal{W}_{\alpha \beta \gamma}=0
$$

A real supervector field $\zeta=\zeta^{B} E_{B}$ is called conformal Killing if

$$
\left[\zeta^{B} \mathcal{D}_{B}+\frac{1}{2} K^{\beta \gamma}[\zeta] M_{\beta \gamma}, \mathcal{D}_{A}\right]+\delta_{\Sigma[\zeta]} \mathcal{D}_{A}=0,
$$

for some Lorentz $\left(K^{\beta \gamma}[\zeta]\right)$ and super-Weyl $(\Sigma[\zeta])$ parameters. This equation implies that $\zeta^{\beta \gamma}$ is the only independent transformation parameter,

$$
\zeta^{\beta}=\frac{\mathrm{i}}{6} \mathcal{D}_{\gamma} \zeta^{\beta \gamma}, \quad K_{\beta \gamma}[\zeta]=2 \mathcal{D}_{(\beta} \zeta_{\gamma)}-2 \mathcal{S} \zeta_{\beta \gamma}, \quad \Sigma[\zeta]=\mathcal{D}_{\beta} \zeta^{\beta}=\frac{1}{3} \mathcal{D}_{b} \zeta^{b}
$$

and it obeys the superconformal Killing equation

$$
\mathcal{D}_{(\alpha} \zeta_{\beta \gamma)}=0 \quad \Longrightarrow \quad \mathcal{D}_{(\alpha \beta} \zeta_{\gamma \delta)}=0 \quad \Longleftrightarrow \mathcal{D}^{a} \zeta^{b}+\mathcal{D}^{b} \zeta^{a}=2 \eta^{a b} \Sigma[\zeta]
$$

The conformal Killing supervector fields of $\mathcal{M}^{3 \mid 2}$ span the conformal superalgebra of $\mathcal{M}^{3 \mid 2}$, which is isomorphic to $\mathfrak{o s p}(1 \mid 4, \mathbb{R})$. A primary tensor superfield $\Phi$ (with suppressed indices) possesses the following superconformal transformation law

$$
-\delta_{\zeta} \Phi=\left(\zeta^{B} \mathcal{D}_{B}+\frac{1}{2} K^{\beta \gamma}[\zeta] M_{\beta \gamma}+d_{\Phi} \Sigma[\zeta]\right) \Phi,
$$

where $d_{\Phi}$ is the dimension of $\Phi$. It should be pointed out that the conformal Killing supervector field $\zeta^{B}$ contains two independent component fields, $\zeta^{b} \mid$ and $\zeta^{\beta} \mid{ }^{16}$

Let us fix a positive integer $n \geq 1$. The conformal gauge supermultiplet $H_{\alpha(n)}$ is a real primary superfield of dimension

$$
d_{H_{\alpha(n)}}=1-\frac{n}{2}
$$

with the gauge transformation law

$$
\delta_{\Lambda} H_{\alpha(n)}=\mathrm{i}^{n} \mathcal{D}_{\left(\alpha_{1}\right.} \Lambda_{\left.\alpha_{2} \ldots \alpha_{n}\right)},
$$

with the gauge parameter $\Lambda_{\alpha(n-1)}$ being real but otherwise unconstrained. In accordance with [42], the super-Cotton tensor $\mathfrak{W}_{\alpha(n)}(H)$ associated with $H_{\alpha(n)}$ is defined to satisfy the following three conditions: (i) it is a primary descendent of $H_{\alpha(n)}$; (ii) it is transverse (or covariantly conserved),

$$
0=\mathcal{D}^{\beta} \mathfrak{W}_{\beta \alpha(n-1)}(H)
$$

(iii) it is invariant under the gauge transformations (4.16),

$$
0=\mathfrak{W}_{\alpha(n)}\left(\delta_{\Lambda} H\right)
$$

\footnotetext{
${ }^{16}$ As usual, the bar-projection of a tensor superfield $V=V(x, \theta)$ (with suppressed indices) is defined by $V|:=V(x, \theta)|_{\theta=0}$.
} 
The conditions (i) and (ii) fix the dimension of $\mathfrak{W}_{\alpha(n)}(H)$ to be

$$
d_{\mathfrak{W}_{\alpha(n)}}=1+\frac{n}{2} .
$$

It was argued in [42] that the supermultiplets $\mathfrak{W}_{\alpha(n)}(H)$ exist for $n \geq 1$ in any conformally flat superspace $\mathcal{M}^{3 \mid 2}$. In terms of conformally covariant derivatives $\nabla_{A}=\left(\nabla_{a}, \nabla_{\alpha}\right)$ [5], the super-Cotton tensors were constructed in [7]. Below we will derive closed-form expressions for $\mathfrak{W}_{\alpha(n)}(H)$ in terms of the Lorentz covariant derivatives $\mathcal{D}_{A}$ for the case $\mathcal{M}^{3 \mid 2}=\operatorname{AdS}^{3 \mid 2}$, i.e. the $\mathcal{N}=1$ AdS superspace.

\section{3 $\mathcal{N}=1$ AdS superspace geometry}

The geometry of $\operatorname{AdS}^{3 / 2}$ is encoded in its covariant derivatives, $\mathcal{D}_{A}$, with the graded commutation relations $[86,89]$

$$
\begin{aligned}
\left\{\mathcal{D}_{\alpha}, \mathcal{D}_{\beta}\right\} & =2 \mathrm{i} \mathcal{D}_{\alpha \beta}-4 \mathrm{i} \mathcal{S} M_{\alpha \beta} \\
{\left[\mathcal{D}_{\alpha \beta}, \mathcal{D}_{\gamma}\right] } & =-2 \mathcal{S} \varepsilon_{\gamma(\alpha} \mathcal{D}_{\beta)} \\
{\left[\mathcal{D}_{\alpha \beta}, \mathcal{D}_{\gamma \delta}\right] } & =4 \mathcal{S}^{2}\left(\varepsilon_{\gamma(\alpha} M_{\beta) \delta}+\varepsilon_{\delta(\alpha} M_{\beta) \gamma}\right),
\end{aligned}
$$

with $\mathcal{S} \neq 0$ a constant real parameter, which may be positive or negative. The two choices, $\mathcal{S}=|\mathcal{S}|$ and $\mathcal{S}=-|\mathcal{S}|$, correspond to the so-called $(1,0)$ and $(0,1)$ AdS superspaces [89], which are different realisations of $\mathcal{N}=1$ AdS superspace. The $(1,0)$ and $(0,1)$ AdS superspaces are naturally embedded in $(1,1)$ AdS superspace $[73,89]$ and are related to each other by a parity transformation. ${ }^{17}$

Isometries of $\operatorname{AdS}^{3 \mid 2}$ are generated by Killing supervector fields $\zeta^{B}$ on $\operatorname{AdS}^{3 \mid 2}$. They are defined to be those conformal Killing supervector fields, eq. (4.13), which obey the additional restriction $\Sigma[\zeta]=0$,

$$
\left[\zeta^{B} \mathcal{D}_{B}+\frac{1}{2} K^{\beta \gamma}[\zeta] M_{\beta \gamma}, \mathcal{D}_{A}\right]=0 .
$$

The important properties of the Killing supervector fields include the following:

$$
\begin{aligned}
\mathcal{D}_{\alpha} \zeta_{\beta \gamma} & =2 \mathrm{i}\left(\varepsilon_{\alpha \beta} \zeta_{\gamma}+\varepsilon_{\alpha \gamma} \zeta_{\beta}\right), \\
\mathcal{D}_{\alpha} \zeta_{\beta} & =\frac{1}{2} K_{\alpha \beta}[\zeta]+\mathcal{S} \zeta_{\alpha \beta}, \\
\mathcal{D}_{\alpha} K_{\beta \gamma}[\zeta] & =4 \mathrm{i} \mathcal{S}\left(\varepsilon_{\alpha \beta} \zeta_{\gamma}+\varepsilon_{\alpha \gamma} \zeta_{\beta}\right),
\end{aligned}
$$

and their corollary

$$
\left(\mathrm{i} \mathcal{D}^{2}+12 \mathcal{S}\right) \zeta_{\alpha}=0
$$

where we have denoted $\mathcal{D}^{2}=\mathcal{D}^{\alpha} \mathcal{D}_{\alpha}$. The relations (4.22) tell us that the $\theta$-dependence of the Killing superfield parameters $\zeta^{B}(x, \theta)$ and $K^{\beta \gamma}(x, \theta)$ in (4.21) is determined by their

\footnotetext{
${ }^{17}$ The only difference between the $(1,0)$ and $(0,1)$ AdS supersymmetry types is the fact that the mass terms in the corresponding Killing spinor equations (4.40) have different signs.
} 
values at $\theta=0$. Given a tensor superfield $\Phi$ on $\operatorname{AdS}^{3 \mid 2}$, its $\operatorname{AdS}$ transformation law is obtained from (4.14) by setting $\Sigma[\zeta]=0$,

$$
-\delta_{\zeta} \Phi=\left(\zeta^{B} \mathcal{D}_{B}+\frac{1}{2} K^{\beta \gamma}[\zeta] M_{\beta \gamma}\right) \Phi .
$$

We recall that the independent component fields of $\zeta^{B}$ are $\zeta^{b} \mid$ and $\zeta^{\beta} \mid$. These components are, respectively, a Killing vector field and a Killing spinor field on $\mathrm{AdS}_{3}$. The equation on the Killing spinor field follows from projecting

$$
\mathcal{D}_{\alpha \beta} \zeta_{\gamma}=-\mathcal{S}\left(\varepsilon_{\gamma \alpha} \zeta_{\beta}+\varepsilon_{\gamma \beta} \zeta_{\alpha}\right) \quad \Longleftrightarrow \mathcal{D}_{a} \zeta_{\beta}=\mathcal{S}\left(\gamma_{a}\right)_{\beta}{ }^{\gamma} \zeta_{\gamma}
$$

which is a simple corollary of (4.22).

There are two independent quadratic Casimir operators ${ }^{18}$ of the $\mathcal{N}=1$ AdS isometry supergroup, $\operatorname{OSp}(1 \mid 2 ; \mathbb{R}) \times \mathrm{SL}(2, \mathbb{R})$, which may be chosen as follows:

$$
\begin{array}{rlrl}
\mathbb{F} & :=-\frac{\mathrm{i}}{2} \mathcal{D}^{2}+2 \mathcal{D}^{\alpha \beta} M_{\alpha \beta}, & & {\left[\mathbb{F}, \mathcal{D}_{A}\right]=0,} \\
\mathbb{Q}:=-\frac{1}{4} \mathcal{D}^{2} \mathcal{D}^{2}+\mathrm{i} \mathcal{S} \mathcal{D}^{2}, & {\left[\mathbb{Q}, \mathcal{D}_{A}\right]=0 .}
\end{array}
$$

The second of these may be expressed in terms of the AdS d'Alembertian, $\square=\mathcal{D}^{a} \mathcal{D}_{a}$, via the relation

$$
-\frac{1}{4} \mathcal{D}^{2} \mathcal{D}^{2}=\square-2 \mathrm{i} \mathcal{S} \mathcal{D}^{2}+2 \mathcal{S} \mathcal{D}^{\alpha \beta} M_{\alpha \beta}-2 \mathcal{S}^{2} M^{\alpha \beta} M_{\alpha \beta}
$$

The fact that they are Casimir operators follows from the useful identities:

$$
\begin{aligned}
\mathcal{D}_{\alpha} \mathcal{D}_{\beta} & =\mathrm{i} \mathcal{D}_{\alpha \beta}-2 \mathrm{i} S M_{\alpha \beta}+\frac{1}{2} \varepsilon_{\alpha \beta} \mathcal{D}^{2}, \\
\mathcal{D}^{\beta} \mathcal{D}_{\alpha} \mathcal{D}_{\beta} & =4 \mathrm{i} S \mathcal{D}_{\alpha}, \quad\left\{\mathcal{D}^{2}, \mathcal{D}_{\alpha}\right\}=4 \mathrm{i} S \mathcal{D}_{\alpha}, \\
\mathcal{D}^{2} \mathcal{D}_{\alpha} & =2 \mathrm{i} \mathcal{S} \mathcal{D}_{\alpha}+2 \mathrm{i} \mathcal{D}_{\alpha \beta} \mathcal{D}^{\beta}-4 \mathrm{i} \mathcal{S} \mathcal{D}^{\beta} M_{\alpha \beta}, \\
{\left[\mathcal{D}_{\alpha} \mathcal{D}_{\beta}, \mathcal{D}^{2}\right] } & =0 \quad \Longrightarrow \quad\left[\mathcal{D}_{\alpha \beta}, \mathcal{D}^{2}\right]=0, \\
{\left[\mathcal{D}_{\alpha}, \square\right] } & =2 \mathcal{S} \mathcal{D}_{\alpha \beta} \mathcal{D}^{\beta}+3 \mathcal{S}^{2} \mathcal{D}_{\alpha} .
\end{aligned}
$$

It is an instructive exercise to check these identities.

\subsection{On-shell massive and partially massless superfields in $\mathrm{AdS}_{3}$}

We define an on-shell real superfield $H_{\alpha(n)}$, with $n \geq 1$, to be one which satisfies the constraints

$$
\begin{aligned}
& 0=\mathcal{D}^{\beta} H_{\alpha(n-1) \beta}, \\
& 0=(\mathbb{F}-M) H_{\alpha(n)},
\end{aligned}
$$

\footnotetext{
${ }^{18}$ For the symplectic groups our notation is $\operatorname{Sp}(2 n, \mathbb{R}) \subset \mathrm{GL}(2 n, \mathbb{R})$, hence $\mathrm{Sp}(2, \mathbb{R}) \cong \mathrm{SL}(2, \mathbb{R}) \cong \mathrm{SU}(1,1)$.
} 
for some mass parameter $M$ which can take any real value, $M \in \mathbb{R}$. The field $H_{\alpha(n)}$ is said to be transverse with pseudo-mass $M$, superspin $n / 2$ and superhelicity $\frac{1}{2}\left(n+\frac{1}{2}\right) \sigma$, where $\sigma=M /|M|$. It can be shown that any superfield satisfying (4.29a) also satisfies

$$
-\frac{\mathrm{i}}{2} \mathcal{D}^{2} H_{\alpha(n)}=\left(\mathcal{D}_{\left(\alpha_{1}\right.}{ }^{\beta}+(n+2) \mathcal{S} \delta_{\left(\alpha_{1}\right.}{ }^{\beta}\right) H_{\left.\alpha_{2} \ldots \alpha_{n}\right) \beta} .
$$

Furthermore, if it satisfies both (4.29a) and (4.29b), then as a consequence we have

$$
-\frac{\mathrm{i}}{2} \mathcal{D}^{2} H_{\alpha(n)}=\frac{1}{2 n+1}(M+2 n(n+2) \mathcal{S}) H_{\alpha(n)} .
$$

From this we can deduce that the second-order wave equation which $H_{\alpha(n)}$ satisfies is

$$
0=\left(\mathbb{Q}-\frac{1}{(2 n+1)^{2}}[M+2 n(n+2) \mathcal{S}][M+2(n-1)(n+1) \mathcal{S}]\right) H_{\alpha(n)} .
$$

The equations (4.29a) and (4.31) were introduced in [90]. In the flat superspace limit, they reduce to the mass-shell equations given in [40].

A novel feature of three-dimensional field theories in AdS superspace is the existence of two distinct types of partially massless superfields. We will say that an on-shell superfield has type A or type B partially massless symmetry if, in addition to (4.29), its pseudo mass satisfies

$$
\begin{aligned}
M \equiv M_{(t, n)}^{(A)}=-2[n(n-2 t)-(t+1)] \mathcal{S}, & & 0 \leq t \leq\lceil n / 2\rceil-1, \\
M \equiv M_{(t, n)}^{(B)}=2[n(n-2 t+1)-(t-1)] \mathcal{S}, & & 1 \leq t \leq\lfloor n / 2\rfloor,
\end{aligned}
$$

respectively. We will refer to these as type $\mathrm{A}$ and type $\mathrm{B}$ pseudo masses, and we say that the corresponding superfield carries super-depth $t$. The latter is an integer whose range of allowed values for each type is specified in (4.33). The relation

$$
M_{(\lceil n / 2\rceil+t-1, n)}^{(A)}=M_{(\lfloor n / 2\rfloor-t+1, n)}^{(B)}
$$

holds between the two types of masses for all $t$. The second order wave equation (4.32) satisfied by partially massless superfields reduces to

$$
0=\left(\mathbb{Q}-\lambda_{(t, n)}^{(A)} \mathcal{S}^{2}\right) H_{\alpha(n)}^{(t, A)}, \quad 0=\left(\mathbb{Q}-\lambda_{(t, n)}^{(B)} \mathcal{S}^{2}\right) H_{\alpha(n)}^{(t, B)},
$$

where the constants

$$
\lambda_{(t, n)}^{(A)}=4 t(t+1), \quad \lambda_{(t, n)}^{(B)}=4(n-t)(n-t+1),
$$

will be referred to as the type A and type B partially massless values.

It may be shown that the system of equations satisfied by on-shell type A and type B partially massless superfields, $H_{\alpha(n)}^{(t, A)}$ and $H_{\alpha(n)}^{(t, B)}$ respectively, admit the gauge symmetries

$$
\begin{aligned}
& \delta_{\Lambda} H_{\alpha(n)}^{(t, A)}=\mathrm{i}^{n} \mathcal{D}_{\left(\alpha_{1} \alpha_{2}\right.} \cdots \mathcal{D}_{\alpha_{2 t-1} \alpha_{2 t}} \mathcal{D}_{\alpha_{2 t+1}} \Lambda_{\left.\alpha_{2 t+2} \ldots \alpha_{n}\right)}, \\
& \delta_{\Lambda} H_{\alpha(n)}^{(t, B)}=\mathcal{D}_{\left(\alpha_{1} \alpha_{2}\right.} \cdots \mathcal{D}_{\alpha_{2 t-1} \alpha_{2 t}} \Lambda_{\left.\alpha_{2 t+1} \ldots \alpha_{n}\right)} .
\end{aligned}
$$


Similar to the non-supersymmetric case (see eq. (2.14)), this is true only if the gauge parameters $\Lambda_{\alpha(n-2 t-1)}$ and $\Lambda_{\alpha(n-2 t)}$ are also on-shell with the same pseudo-mass as their gauge field. We point out that strictly massless superfields $H_{\alpha(n)}$, defined modulo the standard gauge transformations (4.16), correspond to type A partially massless superfields with the minimal super-depth of $t=0$.

\subsection{Component analysis}

In order to make the content of the two types of partially massless supermultiplets more transparent, in this section we study their component structure in more detail.

We start with some general comments. Every Killing supervector field $\zeta^{B}$ on $\operatorname{AdS}^{3 \mid 2}$ can be uniquely decomposed as a sum

$$
\zeta^{B}=\zeta_{(\text {even })}^{B}+\zeta_{(\text {odd })}^{B},
$$

where $\zeta_{(\text {even })}^{B}$ and $\zeta_{(\text {odd })}^{B}$ are even $\left(v^{b}:=\zeta_{(\text {even })}^{b} \mid \neq 0\right.$ and $\left.\zeta_{(\text {even })}^{\beta} \mid=0\right)$ and odd $\left(\zeta_{(\text {odd })}^{b} \mid=0\right.$ and $\left.\epsilon^{\beta}:=\zeta_{(\text {even })}^{\beta} \mid \neq 0\right)$ Killing supervector fields, respectively. Here $v^{b}(x)$ is a Killing vector field on $\mathrm{AdS}_{3}$,

$$
\mathcal{D}^{a} v^{b}+\mathcal{D}^{b} v^{a}=0
$$

and $\epsilon^{\beta}(x)$ is a Killing spinor field on $\mathrm{AdS}_{3}$,

$$
\mathcal{D}_{\alpha \beta} \epsilon_{\gamma}=-\mathcal{S}\left(\varepsilon_{\gamma \alpha} \epsilon_{\beta}+\varepsilon_{\gamma \beta} \epsilon_{\alpha}\right)
$$

Let us consider a tensor superfield $\Phi$ on $\operatorname{AdS}^{3 \mid 2}$ with the transformation law (4.24). Its independent component fields are contained in the set of fields $\varphi=\mathfrak{V} \mid$, where $\mathfrak{V}:=$ $\left\{\Phi, \mathcal{D}_{\alpha} \Phi, \cdots\right\}$. Choosing $\zeta^{B}$ in (4.24) to be $\zeta_{(\text {even })}^{B}$, one observes that the component fields $\varphi$ transform as tensor fields on $\mathrm{AdS}_{3}$,

$$
-\delta_{v} \varphi=\left(v^{b} \mathcal{D}_{b}+\frac{1}{2} K^{\beta \gamma}[v] M_{\beta \gamma}\right) \varphi .
$$

Choosing $\zeta^{B}$ in (4.24) to be $\zeta_{\text {(odd) }}^{B}$, we find that the supersymmetry transformation laws of the component fields are given by ${ }^{19}$

$$
-\delta_{\epsilon} \varphi=\epsilon^{\beta}\left(\mathcal{D}_{\beta} \mathfrak{V}\right) \mid
$$

If $H_{\alpha(n)}$ is an on-shell superfield on $\operatorname{AdS}^{3 \mid 2}$ with pseudo-mass $M$, then it has only two independent component fields, which we define according to

$$
h_{\alpha(n)}:=H_{\alpha(n)}\left|, \quad \psi_{\alpha(n+1)}:=\mathrm{i}^{n+1} \mathcal{D}_{\left(\alpha_{1}\right.} H_{\left.\alpha_{2} \ldots \alpha_{n+1}\right)}\right|=\mathrm{i}^{n+1} \mathcal{D}_{\alpha_{1}} H_{\alpha_{2} \ldots \alpha_{n+1}} \mid .
$$

As a consequence of (4.29a) and the identities (4.28), both component fields are transverse

$$
0=\mathcal{D}^{\beta \gamma} h_{\beta \gamma \alpha(n-2)}, \quad 0=\mathcal{D}^{\beta \gamma} \psi_{\beta \gamma \alpha(n-1)} .
$$

\footnotetext{
${ }^{19}$ To derive this transformation rule, the relation $K_{\alpha \beta}[\zeta]=\frac{1}{2} \mathcal{D}^{\gamma}{ }_{(\alpha} \zeta_{\beta) \gamma}$ may be useful.
} 
To deduce the other first order constraint which they satisfy, the relation

$$
\mathbb{F} \Phi_{\alpha(n)}\left|=\left(\frac{2 n+1}{n} \mathcal{F}+(n+2) \mathcal{S}\right) \Phi_{\alpha(n)}\right|,
$$

which holds for an arbitrary transverse superfield $\Phi_{\alpha(n)}$, is useful. Making use of (4.45), we find that the component fields satisfy

$$
\begin{aligned}
& 0=\left(\mathcal{F}-\frac{n}{2 n+1}[M-(n+2) \mathcal{S}]\right) h_{\alpha(n)}, \\
& 0=\left(\mathcal{F}-\frac{n+1}{2 n+1}[M+(n-1) \mathcal{S}]\right) \psi_{\alpha(n+1)} .
\end{aligned}
$$

The equations (4.44) and (4.46) define on-shell fields in accordance with section 2.2. Finally, making use of (4.42) and (4.29), the supersymmetry transformation laws of the component fields prove to be

$$
\begin{aligned}
-\delta_{\epsilon} h_{\alpha(n)} & =(-\mathrm{i})^{n+1} \epsilon^{\beta} \psi_{\beta \alpha(n)}, \\
-\delta_{\epsilon} \psi_{\alpha(n+1)} & =-\mathrm{i}^{n} \epsilon^{\beta} \mathcal{D}_{\beta \alpha} h_{\alpha(n)}+\frac{\mathrm{i}^{n}}{(2 n+1)}(M+n(4 n+5) \mathcal{S}) \epsilon_{\alpha} h_{\alpha(n)} .
\end{aligned}
$$

Let us now consider the case when the on-shell superfield is type A or type B partially massless with super-depth $t$. Upon substituting $M=M_{(t, n)}^{(A)}$, (4.46) reduces to

$$
0=\left(\mathcal{F}-\rho_{(t, n)}^{(-)}\right) h_{\alpha(n)}, \quad 0=\left(\mathcal{F}-\rho_{(t+1, n+1)}^{(-)}\right) \psi_{\alpha(n+1)} .
$$

On the other-hand, substituting $M=M_{(t, n)}^{(B)}$ we find

$$
0=\left(\mathcal{F}-\rho_{(t, n)}^{(+)}\right) h_{\alpha(n)}, \quad 0=\left(\mathcal{F}-\rho_{(t, n+1)}^{(+)}\right) \psi_{\alpha(n+1)}
$$

We see that the type A supermultiplet $H_{\alpha(n)}^{(t, A)}$ consists of two negative helicity partially massless fields: one with spin $n / 2$ and depth $t$, and the other with spin $(n+1) / 2$ and depth $t+1$. In contrast, the type B supermultiplet $H_{\alpha(n)}^{(t, B)}$ consists of two positive helicity depth- $t$ partially massless fields: one with spin $n / 2$ and the other with spin $(n+1) / 2$.

\subsection{Linearised higher-spin super-Cotton tensors}

In Minkowski superspace, the linearised higher-spin super-Cotton tensor [39, 40] is

$$
\mathfrak{W}_{\alpha_{1} \ldots \alpha_{n}}(H)=\left(-\frac{\mathrm{i}}{2}\right)^{n} D^{\beta_{1}} D_{\left(\alpha_{1}\right.} \ldots D^{\beta_{n}} D_{\left.\alpha_{n}\right)} H_{\beta_{1} \ldots \beta_{n}},
$$

with $D_{A}=\left(\partial_{a}, D_{\alpha}\right)$ being the flat-superspace covariant derivatives. This tensor is invariant under the gauge transformations

$$
\delta_{\Lambda} H_{\alpha(n)}=\mathrm{i}^{n} D_{\left(\alpha_{1}\right.} \Lambda_{\left.\alpha_{2} \ldots \alpha_{n}\right)},
$$


and obeys the conservation identity

$$
D^{\beta} \mathfrak{W}_{\beta \alpha_{1} \ldots \alpha_{n-1}}(H)=0 .
$$

In contrast to the non-supersymmetric case, explicit expressions for the higher-spin super-Cotton tensors are easily obtained in $\mathcal{N}=1$ AdS superspace. In [42], some of the lower rank super-Cotton tensors were derived using the operator

$$
\Delta^{\alpha}{ }_{\beta}:=-\frac{\mathrm{i}}{2} \mathcal{D}^{\alpha} \mathcal{D}_{\beta}-2 \mathcal{S} \delta^{\alpha}{ }_{\beta}
$$

which satisfies the relations

$$
\mathcal{D}^{\beta} \Delta^{\alpha}{ }_{\beta}=0, \quad \Delta^{\alpha}{ }_{\beta} \mathcal{D}_{\alpha}=0 .
$$

To derive the higher-spin super-Cotton tensors, we make use of the following extension of $(4.53)$

$$
\Delta_{[j] \beta}^{\alpha}:=-\frac{\mathrm{i}}{2} \mathcal{D}^{\alpha} \mathcal{D}_{\beta}-2 j \mathcal{S} \delta^{\alpha}{ }_{\beta},
$$

for which (4.53) corresponds to the $j=1$ instance, $\Delta^{\alpha}{ }_{\beta} \equiv \Delta_{[1] \beta}^{\alpha}$. Using the algebra (4.28), it may be shown that they possess the following important properties

$$
\begin{aligned}
{\left[\Delta_{[j]}^{\alpha_{1} \beta_{1}}, \Delta_{[k]}^{\alpha_{2} \beta_{2}}\right] } & =\varepsilon_{\beta_{1} \beta_{2}} \mathcal{S}\left(\mathcal{D}^{\alpha(2)}-2 \mathcal{S} M^{\alpha(2)}\right)-\varepsilon^{\alpha_{1} \alpha_{2}} \mathcal{S}\left(\mathcal{D}_{\beta(2)}-2 \mathcal{S} M_{\beta(2)}\right), \\
\Delta_{[j] \gamma}^{\alpha} \Delta_{[j+1]}^{\beta} \varepsilon^{\delta \gamma} & =j \mathcal{S} \varepsilon^{\alpha \beta}\left(\mathrm{i} \mathcal{D}^{2}+4(j+1) \mathcal{S}\right), \\
\Delta_{[j+1] \gamma}^{\alpha} \Delta_{[j]}^{\beta} \varepsilon_{\alpha \beta} & =j \mathcal{S} \varepsilon_{\gamma \delta}\left(\mathrm{i} \mathcal{D}^{2}+4(j+1) \mathcal{S}\right),
\end{aligned}
$$

for arbitrary integers $j$ and $k$.

In terms of (4.55), the higher-spin super-Cotton tensor takes the remarkably simple form

$$
\begin{aligned}
\mathfrak{W}_{\alpha(n)}(H) & =\Delta_{[1]\left(\alpha_{1}\right.}^{\beta_{1}} \Delta_{[2] \alpha_{2}}^{\beta_{2}} \cdots \Delta_{\left.[n] \alpha_{n}\right)}^{\beta_{n}} H_{\beta(n)} \\
& =\Delta_{[n]\left(\alpha_{1}\right.}^{\beta_{1}} \Delta_{[n-1] \alpha_{2}}^{\beta_{2}} \cdots \Delta_{\left.[1] \alpha_{n}\right)}^{\beta_{n}} H_{\beta(n)} .
\end{aligned}
$$

The equivalence of the two above expressions for $\mathfrak{W}_{\alpha(n)}(H)$ follows from the identity (4.56a). The defining features of the super-Cotton tensors follow immediately from the properties (4.56) of the operators (4.55). In particular, transversality may be shown as follows

$$
\begin{aligned}
\mathcal{D}^{\gamma} \mathfrak{W}_{\gamma \alpha(n-1)}(H) & =\mathcal{D}^{\gamma} \Delta_{[1](\gamma}^{\beta_{1}} \Delta_{[2] \alpha_{1}}^{\beta_{2}} \cdots \Delta_{\left.[n] \alpha_{n-1}\right)}^{\beta_{n}} H_{\beta(n)} \\
& =\frac{1}{n !} \mathcal{D}^{\gamma}\left(\Delta_{[1] \gamma}^{\beta_{1}} \Delta_{[2]}^{\beta_{2}} \cdots \Delta_{[n]}^{\beta_{n} \alpha_{n-1}}+(n !-1) \text { permutations }\right) H_{\beta(n)} .
\end{aligned}
$$

In the last line, all of the $(n !-1)$ permutations may be brought into the same form as the first term using (4.56b). From the first equation in (4.54), it follows that the right hand side of (4.58) vanishes, and hence

$$
0=\mathcal{D}^{\beta} \mathfrak{W}_{\beta \alpha(n-1)}(H)
$$


In a similar vein, its variation under the gauge transformation (4.16) may be computed as follows

$$
\begin{aligned}
\mathfrak{W}_{\alpha(n)}\left(\delta_{\Lambda} H\right) & =\mathrm{i}^{n} \Delta_{[n]\left(\alpha_{1}\right.}^{\beta_{1}} \Delta_{[n-1] \alpha_{2}}^{\beta_{2}} \cdots \Delta_{\left.[1] \alpha_{n}\right)}^{\beta_{n}} \mathcal{D}_{\left(\beta_{1}\right.} \Lambda_{\left.\beta_{2} \ldots \beta_{n}\right)} \\
& =\frac{\mathrm{i}^{n}}{n !} \Delta_{[n]}^{\beta_{1}}\left(\alpha_{1} \Delta_{[n-1]}^{\beta_{2}} \cdots \Delta_{\left.[1] \alpha_{n}\right)}^{\beta_{n}}\left(\mathcal{D}_{\beta_{n}} \Lambda_{\beta_{1} \ldots \beta_{n-1}}+(n !-1) \text { permutations }\right) .\right.
\end{aligned}
$$

This time using $(4.56 \mathrm{c})$, all of the $(n !-1)$ permutations may be brought into the same form as the first term in the second line. From the second identity in (4.54) it follows that the right hand side vanishes,

$$
0=\mathfrak{W}_{\alpha(n)}\left(\delta_{\Lambda} H\right)
$$

and hence $\mathfrak{W}_{\alpha(n)}(H)$ is gauge invariant.

\subsection{Factorisation of the higher-spin super-Cotton tensor}

The gauge invariance and transversality of the higher-spin super-Cotton tensor means that its associated higher-spin Chern-Simons-type functional [7, 42]

$$
S_{\mathrm{SCS}}^{(n)}[H]=-\frac{\mathrm{i}^{n}}{2^{\lfloor n / 2\rfloor+1}} \int \mathrm{d}^{3 \mid 2} z E H^{\alpha(n)} \mathfrak{W}_{\alpha(n)}(H), \quad E^{-1}=\operatorname{Ber}\left(E_{A}{ }^{M}\right)
$$

is manifestly gauge invariant; here we have denoted $\mathrm{d}^{3 \mid 2} z=\mathrm{d}^{3} x \mathrm{~d}^{2} \theta$. In the flat-superspace limit, the action (4.62) reduces to the one given in $[39,40]$. On account of the two equivalent forms of $\mathfrak{W}_{\alpha(n)}(H)$ in (4.57), it is also symmetric in the sense analogous to (2.24). Since $H^{\alpha(n)}$ and $\mathfrak{W}_{\alpha(n)}(H)$ are primary superfields of dimensions (4.15) and (4.19) respectively, the action (4.62) is superconformal.

Similar to the non-supersymmetric case, we may impose the transverse gauge condition

$$
H_{\alpha(n)} \equiv H_{\alpha(n)}^{\mathrm{T}}, \quad 0=\mathcal{D}^{\beta} H_{\beta \alpha(n-1)}^{\mathrm{T}},
$$

under which the super-Cotton tensor takes the form

$$
\begin{aligned}
\mathfrak{W}_{\alpha(n)}\left(H^{\mathrm{T}}\right) & =\frac{1}{(2 n+1)^{n}} \prod_{t=0}^{n-1}\left(\mathbb{F}-M_{(t, n)}^{(A)}\right) H_{\alpha(n)}^{\mathrm{T}} \\
& =\frac{1}{(2 n+1)^{n}} \prod_{t=0}^{\lceil n / 2\rceil-1}\left(\mathbb{F}-M_{(t, n)}^{(A)}\right) \prod_{t=1}^{\lfloor n / 2\rfloor}\left(\mathbb{F}-M_{(t, n)}^{(B)}\right) H_{\alpha(n)}^{\mathrm{T}},
\end{aligned}
$$

where we have used (4.34). We see that on $\operatorname{AdS}^{3 \mid 2}$, the superconformal higher-spin action (4.62) factorises into first-order differential operators involving all of the type A and type B partial pseudo-mass values.

Finally we note that, in any conformally flat superspace, it is also true that the vanishing of the higher-spin Super-Cotton tensor $\mathfrak{W}_{\alpha(n)}(H)$ is a necessary and sufficient condition for $H_{\alpha(n)}$ to be pure gauge,

$$
\mathfrak{W}_{\alpha(n)}(H)=0 \quad \Longleftrightarrow \quad H_{\alpha(n)}=\mathrm{i}^{n} \mathcal{D}_{\alpha} \Lambda_{\alpha(n-1)},
$$


for some $\Lambda_{\alpha(n-1)}$. This is discussed at the end of appendix $\mathrm{C}$, to where we refer the reader for more details. Therefore, from (4.64), it follows that both type A and B partially-massless superfields do not contain any local propagating degrees of freedom.

\subsection{Massive $\mathcal{N}=1$ gauge actions}

Off-shell $\mathcal{N}=1$ supersymmetric extensions of the new topologically massive higher-spin gauge models (3.1) were also proposed in [42]. For any integer $n \geq 1$, the gauge-invariant action corresponding to the unconstrained prepotential $H_{\alpha(n)}$ may be recast into the form

$$
\mathbb{S}_{\mathrm{NTM}}^{(n)}[H]=-\frac{\mathrm{i}^{n}}{2^{\lfloor n / 2\rfloor+1}} \frac{1}{M} \int \mathrm{d}^{3 \mid 2} z E \mathfrak{W}^{\alpha(n)}(H)(\mathbb{F}-M) H_{\alpha(n)} .
$$

The equation of motion obtained by varying (4.66) with respect to $H_{\alpha(n)}$ is

$$
0=(\mathbb{F}-M) \mathfrak{W}_{\alpha(n)}(H)
$$

For generic $M$, this equation in conjunction with the off-shell conservation identity (4.59) means that the field-strength $\mathfrak{W}_{\alpha(n)}(H)$ itself describes a propagating mode with pseudomass $M$, superspin $n / 2$ and superhelicity $\frac{1}{2}\left(n+\frac{1}{2}\right) \sigma$, where $\sigma=M /|M|$. In the case when $M$ takes on one of the type A or type B partially massless values (4.33), an analysis similar to that in the non-supersymmetric case can be conducted, with the conclusion that there are no local propagating degrees of freedom.

Topologically massive supersymmetric higher-spin actions ${ }^{20}$ involve different massless sectors depending on the value of superspin. For integer superspin, the relevant massless action $^{21}$ is first-order (in vector derivatives) and takes the form [42]

$$
\begin{aligned}
\mathbb{S}_{\mathrm{FO}}^{(2 s)}[H, Y]= & \left(-\frac{1}{2}\right)^{s} \frac{\mathrm{i}}{2} \int \mathrm{d}^{3 \mid 2} z E\left\{H^{\beta \alpha(2 s-1)} \mathcal{D}^{\gamma} \mathcal{D}_{\beta} H_{\gamma \alpha(2 s-1)}\right. \\
& +2 \mathrm{i}(2 s-1) Y^{\alpha(2 s-2)} \mathcal{D}^{\beta(2)} H_{\beta(2) \alpha(2 s-2)} \\
& +(2 s-1)\left(Y^{\alpha(2 s-2)} \mathcal{D}^{2} Y_{\alpha(2 s-2)}+(2 s-2) \mathcal{D}_{\beta} Y^{\beta \alpha(2 s-3)} \mathcal{D}^{\gamma} Y_{\gamma \alpha(2 s-3)}\right) \\
& \left.-4 \mathcal{S} \mathrm{i}\left(H^{\alpha(2 s)} H_{\alpha(2 s)}+2 s(2 s-1) Y^{\alpha(2 s-2)} Y_{\alpha(2 s-2)}\right)\right\} .
\end{aligned}
$$

It is invariant under the gauge transformations

$$
\begin{aligned}
\delta_{\Lambda} H_{\alpha(2 s)} & =\mathcal{D}_{\alpha} \Lambda_{\alpha(2 s-1)}, \\
\delta_{\Lambda} Y_{\alpha(2 s-2)} & =\frac{1}{2 s} \mathcal{D}^{\beta} \Lambda_{\beta \alpha(2 s-2)} .
\end{aligned}
$$

\footnotetext{
${ }^{20}$ These are higher-spin extensions of topologically massive $\mathcal{N}=1$ supergravity [48].

${ }^{21}$ There is another massless action of this type, where the superfield $H_{\alpha(2 s)}$ appears in the action with a first-order kinetic operator. However, the gauge transformations are of type B with $t=1, \delta_{\Lambda} H_{\alpha(2 s)}=$ $\mathcal{D}_{\alpha(2)} \Lambda_{\alpha(2 s-2)}$. The corresponding action has been derived recently in [73].
} 
For half-integer superspin, the relevant massless action is second-order and takes the form $[42]$

$$
\begin{aligned}
& \mathbb{S}_{\mathrm{SO}}^{(2 s+1)}[H, Y]=\left(-\frac{1}{2}\right)^{s} \int \mathrm{d}^{3 \mid 2} z E\left\{-\frac{\mathrm{i}}{2} H^{\alpha(2 s+1)} \mathbb{Q} H_{\alpha(2 s+1)}-\frac{\mathrm{i}}{8} \mathcal{D}_{\beta} H^{\beta \alpha(2 s)} \mathcal{D}^{2} \mathcal{D}^{\gamma} H_{\gamma \alpha(2 s)}\right. \\
& +\frac{\mathrm{i}}{4} s \mathcal{D}_{\beta \gamma} H^{\beta \gamma \alpha(2 s-1)} \mathcal{D}^{\rho \lambda} H_{\rho \lambda \alpha(2 s-1)}-\frac{1}{2}(2 s-1) Y^{\alpha(2 s-2)} \mathcal{D}^{\beta \gamma} \mathcal{D}^{\delta} H_{\beta \gamma \delta \alpha(2 s-2)} \\
& +\frac{\mathrm{i}}{2}(2 s-1)\left[Y^{\alpha(2 s-2)} \mathcal{D}^{2} Y_{\alpha(2 s-2)}-\frac{s-1}{s} \mathcal{D}_{\beta} Y^{\beta \alpha(2 s-3)} \mathcal{D}^{\gamma} Y_{\gamma \alpha(2 s-3)}\right] \\
& +\mathrm{i} s \mathcal{S} H^{\beta \alpha(2 s)} \mathcal{D}_{\beta}^{\gamma} H_{\gamma \alpha(2 s)}+\frac{1}{2}(s+1) \mathcal{S} H^{\alpha(2 s+1)} \mathcal{D}^{2} H_{\alpha(2 s+1)} \\
& \left.+\mathrm{i} s(2 s-3) \mathcal{S}^{2} H^{\alpha(2 s+1)} H_{\alpha(2 s+1)}+\frac{(2 s-1)\left(s^{2}-3 s-2\right)}{s} \mathcal{S} Y^{\alpha(2 s-2)} Y_{\alpha(2 s-2)}\right\}
\end{aligned}
$$

and it is invariant under the gauge transformations

$$
\begin{aligned}
\delta_{\Lambda} H_{\alpha(2 s+1)} & =\mathrm{i} \mathcal{D}_{\alpha} \Lambda_{\alpha(2 s)} \\
\delta_{\Lambda} Y_{\alpha(2 s-2)} & =\frac{s}{2 s+1} \mathcal{D}^{\beta(2)} \Lambda_{\beta(2) \alpha(2 s-2)} .
\end{aligned}
$$

The corresponding massive gauge-invariant actions for integer and half-integer superspin are

$$
\begin{aligned}
\mathbb{S}_{\mathrm{TM}}^{(2 s)}[H, Y] & =\mathbb{S}_{\mathrm{SCS}}^{(2 s)}[H]+\boldsymbol{\mu}(M, s) \mathbb{S}_{\mathrm{FO}}^{(2 s)}[H, Y], \\
\mathbb{S}_{\mathrm{TM}}^{(2 s+1)}[H, Y] & =\mathbb{S}_{\mathrm{SCS}}^{(2 s+1)}[H]+\boldsymbol{\nu}(M, s) \mathbb{S}_{\mathrm{SO}}^{(2 s+1)}[H, Y] .
\end{aligned}
$$

The coupling constants $\boldsymbol{\mu}(M, s)$ and $\boldsymbol{\nu}(M, s)$ both have mass dimension $2 s-1$, and are functions of $s$ and some parameter $M$ with mass dimension one. Their explicit form in terms of these quantities may be determined by requiring that any $H_{\alpha(n)}$ satisfying (4.29) is a particular solution to the resulting field equations, as in the non-supersymmetric case. We expect that both $\boldsymbol{\mu}$ and $\boldsymbol{\nu}$ will vanish at the type A and type B partially massless points (4.33), where the models do not describe any local propagating degrees of freedom.

The above massive gauge-invariant actions are manifestly supersymmetric, that is the $\mathcal{N}=1$ AdS supersymmetry is realised off-shell. It is worth pointing out that there also exists an on-shell construction of gauge-invariant Lagrangian formulations for massive higher-spin $\mathcal{N}=1$ supermultiplets in $\mathbb{R}^{2,1}$ and $\mathrm{AdS}_{3}$ [91-93], extending previous works on the non-supersymmetric cases $[65,66]$. These frame-like formulations are based on the gauge-invariant approach to the dynamics of massive higher-spin fields proposed by Zinoviev [62, 94] and Metsaev [63].

\section{Discussion}

In this paper we have derived closed-form expressions for the Cotton tensors $\mathfrak{C}_{\alpha(n)}(h)$ associated with the conformal higher-spin gauge fields $h_{\alpha(n)}$ in $\mathrm{AdS}_{3}$. In the fermionic (odd $n$ ) and bosonic (even $n$ ) cases, they are given by (2.22a) and (2.22b) respectively. Their properties proved to greatly facilitate the analysis of the on-shell dynamics of topologically 
massive higher-spin gauge models (3.15), and their new variant (3.1). In particular, it was shown that these models describe a propagating mode with pseudo mass $\rho$ and helicity $\operatorname{sgn}(\rho) n / 2$, except at the partially massless points (2.9), where they describe only pure gauge degrees of freedom.

In the transverse gauge, the Cotton tensors were demonstrated to factorise into products of second-order operators (2.26) involving all partially massless values and, equivalently, into products of first-order operators (2.28) involving all depth- $t$ pseudo-masses (with both signs of helicity). This in turn demonstrates that the action (2.23) for conformal higher-spin gauge fields factorises, on an $\mathrm{AdS}_{3}$ background, into wave operators associated with partially massless fields. This is in line with earlier results obtained in even dimensions [20, 21, 54, 95-101].

In Minkowski space, it is known [102] that the higher-spin Cotton tensors are proportional to certain linear combinations of the positive and negative helicity spin-projection operators. The latter are the three-dimensional analogues of the Behrends-Fronsdal traceless and transverse (TT) projectors. Additionally, recent studies [101] in $\mathrm{AdS}_{4}$ have revealed that the poles of the corresponding TT projectors are intimately related to partially massless fields. This relation renders the factorisation properties of conformal higher-spin wave operators in $\mathrm{AdS}_{4}$ transparent. It would be an interesting problem to construct the TT projectors in $\mathrm{AdS}_{3}$ and investigate whether similar relations hold.

In this paper we have also provided $\mathcal{N}=1 \mathrm{AdS}_{3}$ superspace extensions to the non-supersymmetric results described above. Specifically, the remarkably simple expression (4.57) for the higher-spin super-Cotton tensor $\mathfrak{W}_{\alpha(n)}(H)$ was derived. In the transverse gauge, it was shown that $\mathfrak{W}_{\alpha(n)}(H)$ factorises into first-order wave operators (4.64) associated with the so-called type A and type B partially massless superfields. Similar remarks regarding the factorisation of the superconformal higher-spin gauge models (4.62) in an $\mathrm{AdS}_{3}$ superspace background follow. The fact that there are two types of partially massless supermultiplets, possessing gauge symmetries (4.37a) and (4.37b), is a novel feature of three dimensions, and did not occur in four dimensions [103].

Finally, it would be interesting to obtain $\mathcal{N}$-extended analogues of the above results, as well as the corresponding $\mathrm{AdS}_{3}$ superprojectors along the lines of $[44,103]$.

\section{Acknowledgments}

SMK is grateful to Ulf Lindström and Gabriele Tartaglino-Mazzucchelli for useful discussions. The authors are grateful to Daniel Hutchings for pointing out some notational inconsistencies and misprints in the manuscript. The work of SMK is supported in part by the Australian Research Council, project No. DP200101944. The work of MP is supported by the Hackett Postgraduate Scholarship UWA, under the Australian Government Research Training Program.

\section{A Two-component spinor toolkit}

We follow the notation and conventions adopted in [77]. In particular, the Minkowski metric is $\eta_{a b}=\operatorname{diag}(-1,1,1)$. The spinor indices are raised and lowered using the $\operatorname{SL}(2, \mathbb{R})$ 
invariant tensors

$$
\varepsilon_{\alpha \beta}=\left(\begin{array}{cc}
0 & -1 \\
1 & 0
\end{array}\right), \quad \varepsilon^{\alpha \beta}=\left(\begin{array}{cc}
0 & 1 \\
-1 & 0
\end{array}\right), \quad \varepsilon^{\alpha \gamma} \varepsilon_{\gamma \beta}=\delta_{\beta}^{\alpha}
$$

by the standard rule:

$$
\psi^{\alpha}=\varepsilon^{\alpha \beta} \psi_{\beta}, \quad \psi_{\alpha}=\varepsilon_{\alpha \beta} \psi^{\beta}
$$

We make use of real gamma-matrices, $\gamma_{a}:=\left(\left(\gamma_{a}\right)_{\alpha}{ }^{\beta}\right)$, which obey the algebra

$$
\gamma_{a} \gamma_{b}=\eta_{a b} \mathbb{1}+\varepsilon_{a b c} \gamma^{c}
$$

where the Levi-Civita tensor is normalised as $\varepsilon^{012}=-\varepsilon_{012}=1$. Given a three-vector $V_{a}$, it can be equivalently described by a symmetric second-rank spinor $V_{\alpha \beta}$ defined as

$$
V_{\alpha \beta}:=\left(\gamma^{a}\right)_{\alpha \beta} V_{a}=V_{\beta \alpha}, \quad V_{a}=-\frac{1}{2}\left(\gamma_{a}\right)^{\alpha \beta} V_{\alpha \beta}
$$

Any antisymmetric tensor $F_{a b}=-F_{b a}$ is Hodge-dual to a three-vector $F_{a}$, specifically

$$
F_{a}=\frac{1}{2} \varepsilon_{a b c} F^{b c}, \quad F_{a b}=-\varepsilon_{a b c} F^{c} .
$$

Then, the symmetric spinor $F_{\alpha \beta}=F_{\beta \alpha}$, which is associated with $F_{a}$, can equivalently be defined in terms of $F_{a b}$ :

$$
F_{\alpha \beta}:=\left(\gamma^{a}\right)_{\alpha \beta} F_{a}=\frac{1}{2}\left(\gamma^{a}\right)_{\alpha \beta} \varepsilon_{a b c} F^{b c}
$$

These three algebraic objects, $F_{a}, F_{a b}$ and $F_{\alpha \beta}$, are in one-to-one correspondence to each other, $F_{a} \leftrightarrow F_{a b} \leftrightarrow F_{\alpha \beta}$. The corresponding inner products are related to each other as follows:

$$
-F^{a} G_{a}=\frac{1}{2} F^{a b} G_{a b}=\frac{1}{2} F^{\alpha \beta} G_{\alpha \beta} .
$$

The Lorentz generators with two vector indices $\left(M_{a b}=-M_{b a}\right)$, one vector index $\left(M_{a}\right)$ and two spinor indices $\left(M_{\alpha \beta}=M_{\beta \alpha}\right)$ are related to each other by the rules: $M_{a}=\frac{1}{2} \varepsilon_{a b c} M^{b c}$ and $M_{\alpha \beta}=\left(\gamma^{a}\right)_{\alpha \beta} M_{a}$. These generators act on a vector $V_{c}$ and a spinor $\Psi_{\gamma}$ as follows:

$$
M_{a b} V_{c}=2 \eta_{c[a} V_{b]}, \quad M_{\alpha \beta} \Psi_{\gamma}=\varepsilon_{\gamma(\alpha} \Psi_{\beta)} .
$$

The following identities hold:

$$
\begin{aligned}
M_{\alpha_{1}}{ }^{\beta} \Phi_{\beta \alpha_{2} \ldots \alpha_{n}} & =-\frac{1}{2}(n+2) \Phi_{\alpha(n)}, \\
M^{\beta \gamma} M_{\beta \gamma} \Phi_{\alpha(n)} & =-\frac{1}{2} n(n+2) \Phi_{\alpha(n)} .
\end{aligned}
$$




\section{B Generating function formalism}

In order to easily prove the defining properties of the higher-spin Cotton tensors, it will be useful to reformulate the problem into one in terms of homogeneous polynomials. This framework is also sometimes referred to as the generating function formalism.

Associated with a symmetric rank- $n$ spinor field $\phi_{\alpha(n)}:=\phi_{\alpha_{1} \ldots \alpha_{n}}=\phi_{\left(\alpha_{1} \ldots \alpha_{n}\right)}$ is a homogeneous polynomial $\phi_{(n)}(\Upsilon)$ of degree $n$ defined by

$$
\phi_{(n)}:=\Upsilon^{\alpha_{1}} \ldots \Upsilon^{\alpha_{n}} \phi_{\alpha_{1} \ldots \alpha_{n}},
$$

where the auxiliary variables $\Upsilon^{\alpha}$ are chosen to be commuting, hence $\Upsilon^{\alpha} \Upsilon_{\alpha}:=\Upsilon^{\alpha} \varepsilon_{\alpha \beta} \Upsilon^{\beta}=$ 0 . The correspondence $\phi_{\alpha(n)} \rightarrow \phi_{(n)}$ is one-to-one. The linear space of such polynomials will be denoted $\mathcal{H}_{(n)}$.

Introducing the auxiliary derivative $\partial_{\alpha}:=\frac{\partial}{\partial \Upsilon^{\alpha}}$, whose index is raised according to the usual rule $\partial^{\alpha}:=\varepsilon^{\alpha \beta} \partial_{\beta}$, we define the AdS differential operators

$$
\mathcal{D}_{(2)}:=\Upsilon^{\alpha} \Upsilon^{\beta} \mathcal{D}_{\alpha \beta}, \quad \mathcal{D}_{(0)}:=\Upsilon^{\alpha} \mathcal{D}_{\alpha}^{\beta} \partial_{\beta}, \quad \mathcal{D}_{(-2)}:=\mathcal{D}^{\alpha \beta} \partial_{\alpha} \partial_{\beta}
$$

They raise the degree of homogeneity of any polynomial on which they act by 2,0 and -2 respectively. Amongst themselves, they may be shown to satisfy the algebra ${ }^{22}$

$$
\begin{aligned}
{\left[\mathcal{D}_{(2)}, \mathcal{D}_{(-2)}\right] } & =4\left(\boldsymbol{\Upsilon}_{(0)}+1\right) \square+8 \mathcal{S}^{2} \boldsymbol{M}_{(0)}\left(\boldsymbol{\Upsilon}_{(0)}+1\right) \\
{\left[\mathcal{D}_{(2)}, \mathcal{D}_{(0)}\right] } & =4 \mathcal{S}^{2} \boldsymbol{M}_{(2)}\left(\boldsymbol{\Upsilon}_{(0)}+2\right) \\
{\left[\mathcal{D}_{(-2)}, \mathcal{D}_{(0)}\right] } & =-4 \mathcal{S}^{2} \boldsymbol{M}_{(-2)}\left(\boldsymbol{\Upsilon}_{(0)}+2\right)
\end{aligned}
$$

where we have made use of the definitions ${ }^{23}$

$$
\begin{aligned}
\boldsymbol{\Upsilon}_{(0)} & :=\Upsilon^{\alpha} \partial_{\alpha}, & \boldsymbol{\Upsilon}_{(0)} \phi_{(n)} & =n \phi_{(n)}, \\
\boldsymbol{M}_{(0)} & :=\Upsilon^{\alpha} M_{\alpha}^{\beta} \partial_{\beta}, & \boldsymbol{M}_{(0)} \phi_{(n)} & =-\frac{1}{2} n(n+2) \phi_{(n)}, \\
\boldsymbol{M}_{(2)} & :=\Upsilon^{\alpha} \Upsilon^{\beta} M_{\alpha \beta}, & \boldsymbol{M}_{(2)} \phi_{(n)} & =0, \\
\boldsymbol{M}_{(-2)} & :=M^{\alpha \beta} \partial_{\alpha} \partial_{\beta}, & \boldsymbol{M}_{(-2)} \phi_{(n)} & =0 .
\end{aligned}
$$

Using the above identities it is possible to show, via induction on $t$, that for any $\phi_{(n)} \in \mathcal{H}_{(n)}$ the following relations hold

$$
\begin{aligned}
& {\left[\mathcal{D}_{(2)}, \mathcal{D}_{(-2)}^{t}\right] \phi_{(n)}=4 t(n-t+2)\left(\mathcal{Q}-\tau_{(t, n+2)} \mathcal{S}^{2}\right) \mathcal{D}_{(-2)}^{t-1} \phi_{(n)}} \\
& {\left[\mathcal{D}_{(-2)}, \mathcal{D}_{(2)}^{t}\right] \phi_{(n)}=-4 t(n+t)\left(\mathcal{Q}-\tau_{(t, n+2 t)} \mathcal{S}^{2}\right) \mathcal{D}_{(2)}^{t-1} \phi_{(n)}}
\end{aligned}
$$

Here $\mathcal{Q}$ is the quadratic Casimir $(2.2 \mathrm{~b})$ and $\tau_{(t, n)}$ are the partially massless values (2.11).

\footnotetext{
${ }^{22}$ The auxiliary variables $\Upsilon^{\alpha}$ are defined to be inert with respect to the Lorentz generators. Alternatively, one could define their action on any $\phi_{(n)} \in \mathcal{H}_{(n)}$ to be $M_{\alpha \beta} \phi_{(n)}:=-\Upsilon_{(\alpha} \partial_{\beta)} \phi_{(n)}$.

${ }^{23}$ The identities (B.4c) and (B.4d) imply that the commutators (B.3b) and (B.3c) vanish on $\mathcal{H}_{(n)}$. This is not surprising given that on $\mathcal{H}_{(n)}$, the operator $\mathcal{D}_{(0)}$ can be identified with the quadratic Casimir $\mathcal{F}$.
} 


\section{Gauge completeness}

In this appendix we sketch a proof for the following result: in a conformally flat background $\mathcal{M}^{3}$, the Cotton tensor $\mathfrak{C}_{\alpha(n)}(h)$ vanishes if and only if the gauge field $h_{\alpha(n)}$ is pure gauge,

$$
\mathfrak{C}_{\alpha(n)}(h)=0 \quad \Longleftrightarrow \quad h_{\alpha(n)}=\mathcal{D}_{\alpha(2)} \xi_{\alpha(n-2)},
$$

for some $\xi_{\alpha(n-2)}$. In the case of Minkowski space, this theorem was proved in [36, 43]. Here we start by giving an alternative derivation in Minkowski space,

$$
\mathfrak{C}_{\alpha(n)}(h)=0 \quad \Longleftrightarrow \quad h_{\alpha(n)}=\partial_{\alpha(2)} \xi_{\alpha(n-2)} .
$$

The Cotton tensor (1.12) is invariant under the gauge transformations

$$
\delta_{\xi} h_{\alpha(n)}=\partial_{\alpha(2)} \xi_{\alpha(n-2)} .
$$

This gauge freedom allows us to choose the gauge

$$
\partial^{\beta(2)} h_{\beta(2) \alpha(n-2)}=0,
$$

in which the Cotton tensor takes the form [42]

$$
\begin{aligned}
\mathfrak{C}_{\alpha(2 s)}(h) & =\square^{s-1} \partial^{\beta}{ }_{\left(\alpha_{1}\right.} h_{\left.\alpha_{2} \ldots \alpha_{2 s}\right) \beta}=\square^{s-1} \partial^{\beta}{ }_{\alpha_{1}} h_{\alpha_{2} \ldots \alpha_{2 s} \beta}, \\
\mathfrak{C}_{\alpha(2 s+1)}(h) & =\square^{s} h_{\alpha(2 s+1)} .
\end{aligned}
$$

Now suppose $\mathfrak{C}_{\alpha(n)}(h)=0$. We assume $h_{\alpha(n)}$ to decrease at infinity, such that its Fourier transform is well defined. Then it follows that for $n>2$ the prepotential obeys the wave equation

$$
\square h_{\alpha(n)}=0,
$$

in addition to the transverse condition (C.4). The equations (C.4) and (C.6) are invariant under a restricted class of gauge transformations (C.3) such that the gauge parameter $\xi_{\alpha(n-2)}$ is constrained by

$$
\partial^{\beta(2)} \xi_{\beta(2) \alpha(n-4)}=0, \quad \square \xi_{\alpha(n-2)}=0 .
$$

This residual gauge freedom suffices to gauge $h_{\alpha(n)}$ away, which implies (C.2). The case $n=2$ is special since the condition $\mathfrak{C}_{\alpha(2)}(h)=0$ gives the first-order equation

$$
\partial^{\beta}{ }_{\left(\alpha_{1}\right.} h_{\left.\alpha_{2}\right) \beta}=\partial^{\beta}{ }_{\alpha_{1}} h_{\alpha_{2} \beta}=0 \quad \Longrightarrow \quad \square h_{\alpha(2)}=0,
$$

and thus the vector field $h_{\alpha(2)}$ has a single independent massless component. The residual gauge freedom is described by a parameter $\xi$ constrained by $\square \xi=0$. This gauge freedom allows us to gauge away $h_{\alpha(2)}$.

To complete the proof of (C.1), it remains to point out that both equations in (C.1) are invariant under Weyl transformations in curved space

$$
\mathcal{D}_{a} \rightarrow \mathcal{D}_{a}^{\prime}=\mathrm{e}^{\sigma}\left(\mathcal{D}_{a}+\mathcal{D}^{b} \sigma M_{b a}\right)
$$


provided $h_{\alpha(n)}$ and $\xi_{\alpha(n-2)}$ are primary fields of dimension $(2-n / 2)$ and $(1-n / 2)$, respectively. As discussed in [42], the Weyl transformation law of $h_{\alpha(n)}$ implies that $\mathfrak{C}_{\alpha(n)}(h)$ is a primary field of dimension (1.10).

Yet another way to arrive at the result (C.2) in Minkowski space is by using the 3D spin-projection operators of [102]. The latter are defined according to

$$
\Pi_{\alpha}^{( \pm) \beta}=\frac{1}{2}\left(\delta_{\alpha}^{\beta} \pm \frac{\partial_{\alpha}^{\beta}}{\sqrt{\square}}\right), \quad \Pi_{\alpha(n)}^{( \pm n) \beta(n)}=\Pi_{\left(\alpha_{1}\right.}^{( \pm) \beta_{1}} \ldots \Pi_{\left.\alpha_{n}\right)}^{( \pm) \beta_{n}},
$$

and satisfy the relations

$$
\Pi^{(+n)} \Pi^{(+n)}=\Pi^{(+n)}, \quad \Pi^{(-n)} \Pi^{(-n)}=\Pi^{(-n)}, \quad \Pi^{(+n)} \Pi^{(-n)}=0 .
$$

Let us define the following combinations

$$
\Pi_{\perp}^{[n]}:=\Pi^{(n)}+\Pi^{(-n)}, \quad \Pi_{\|}^{[n]}:=\mathbb{1}-\Pi_{\perp}^{(n)} .
$$

It is clear that they resolve the identity, $\mathbb{1}=\Pi_{\perp}^{[n]}+\Pi_{\|}^{[n]}$, and are orthogonal projectors

$$
\Pi_{\perp}^{[n]} \Pi_{\perp}^{[n]}=\Pi_{\perp}^{[n]}, \quad \Pi_{\|}^{[n]} \Pi_{\|}^{[n]}=\Pi_{\|}^{[n]}, \quad \Pi_{\perp}^{[n]} \Pi_{\|}^{[n]}=0 .
$$

Furthermore, $\Pi_{\perp}^{[n]}$ projects a field $h_{\alpha(n)}$ onto its transverse component,

$$
h_{\alpha(n)}^{\perp} \equiv \Pi_{\perp}^{[n]} h_{\alpha(n)}, \quad 0=\partial^{\beta(2)} h_{\alpha(n-2) \beta(2)}^{\perp},
$$

whilst $\Pi_{\|}^{[n]}$ projects onto its longitudinal (pure gauge) component,

$$
h_{\alpha(n)}^{\|} \equiv \Pi_{\|}^{[n]} h_{\alpha(n)}, \quad h_{\alpha(n)}^{\|}=\partial_{\alpha(2)} \xi_{\alpha(n-2)},
$$

for some $\xi_{\alpha(n-2)}$. It follows that any unconstrained field $h_{\alpha(n)}$ may be decomposed according to

$$
h_{\alpha(n)}=h_{\alpha(n)}^{\perp}+\partial_{\alpha(2)} \xi_{\alpha(n-2)} .
$$

To arrive at the conclusion (C.2), it remains to recall that the higher-spin Cotton tensors can be expressed as

$$
\mathfrak{C}_{\alpha(2 s)}(h)=\square^{s-1} \Pi_{\perp}^{[2 s]} \partial_{\alpha}{ }^{\beta} h_{\alpha(2 s-1) \beta}, \quad \mathfrak{C}_{\alpha(2 s+1)}(h)=\square^{s} \Pi_{\perp}^{[2 s+1]} h_{\alpha(2 s+1)} .
$$

These relations imply that

$$
h_{\alpha(2 s)}^{\perp}=\square^{-s} \partial_{\alpha}{ }^{\beta} \mathfrak{C}_{\alpha(2 s-1) \beta}(h), \quad h_{\alpha(2 s+1)}^{\perp}=\square^{-s} \mathfrak{C}_{\alpha(2 s+1)}(h),
$$

which completes the proof of (C.2).

In [44], the superprojectors in $\mathcal{N}$-extended Minkowski superspace were derived, and expressions for the corresponding higher-spin linearised super-Cotton tensors were given. The above argument may be replicated in a straightforward manner using the results of [44]. The conclusion is that, in any conformally-flat superspace, a necessary and sufficient condition for a prepotential to be pure gauge is if its super-Cotton tensor vanishes. 
Open Access. This article is distributed under the terms of the Creative Commons Attribution License (CC-BY 4.0), which permits any use, distribution and reproduction in any medium, provided the original author(s) and source are credited.

\section{References}

[1] L.P. Eisenhart, Riemannian Geometry, Princeton University Press, Princeton NJ U.S.A. (1926).

[2] M. Kaku, P.K. Townsend and P. van Nieuwenhuizen, Gauge Theory of the Conformal and Superconformal Group, Phys. Lett. B 69 (1977) 304 [InSPIRE].

[3] M. Kaku, P.K. Townsend and P. van Nieuwenhuizen, Properties of Conformal Supergravity, Phys. Rev. D 17 (1978) 3179 [InSPIRE].

[4] D. Butter, $N=1$ Conformal Superspace in Four Dimensions, Annals Phys. 325 (2010) 1026 [arXiv:0906.4399] [INSPIRE].

[5] D. Butter, S.M. Kuzenko, J. Novak and G. Tartaglino-Mazzucchelli, Conformal supergravity in three dimensions: New off-shell formulation, JHEP 09 (2013) 072 [arXiv:1305.3132] [INSPIRE].

[6] D. Butter, S.M. Kuzenko, J. Novak and S. Theisen, Invariants for minimal conformal supergravity in six dimensions, JHEP 12 (2016) 072 [arXiv:1606.02921] [INSPIRE].

[7] S.M. Kuzenko and M. Ponds, Conformal geometry and (super)conformal higher-spin gauge theories, JHEP 05 (2019) 113 [arXiv: 1902.08010] [INSPIRE].

[8] E.S. Fradkin and A.A. Tseytlin, Conformal supergravity, Phys. Rept. 119 (1985) 233 [INSPIRE].

[9] E.S. Fradkin and V.Y. Linetsky, A Superconformal Theory of Massless Higher Spin Fields in $D=(2+1)$, Annals Phys. 198 (1990) 293 [Mod. Phys. Lett. A 4 (1989) 731] [InSPIRE].

[10] C.N. Pope and P.K. Townsend, Conformal Higher Spin in $(2+1)$-dimensions, Phys. Lett. B 225 (1989) 245 [INSPIRE].

[11] E.S. Fradkin and V.Y. Linetsky, Cubic Interaction in Conformal Theory of Integer Higher Spin Fields in Four-dimensional Space-time, Phys. Lett. B 231 (1989) 97 [InSPIRE].

[12] E.S. Fradkin and V.Y. Linetsky, Superconformal Higher Spin Theory in the Cubic Approximation, Nucl. Phys. B 350 (1991) 274 [INSPIRE].

[13] A.A. Tseytlin, On limits of superstring in $A d S_{5} \times S^{5}$, Theor. Math. Phys. 133 (2002) 1376 [Teor. Mat. Fiz. 133 (2002) 69] [hep-th/0201112] [INSPIRE].

[14] A.Y. Segal, Conformal higher spin theory, Nucl. Phys. B 664 (2003) 59 [hep-th/0207212] [INSPIRE].

[15] R. Marnelius, Lagrangian conformal higher spin theory, arXiv:0805.4686 [INSPIRE].

[16] R.R. Metsaev, Gauge invariant two-point vertices of shadow fields, AdS/CFT, and conformal fields, Phys. Rev. D 81 (2010) 106002 [arXiv:0907.4678] [INSPIRE].

[17] M.A. Vasiliev, Bosonic conformal higher-spin fields of any symmetry, Nucl. Phys. B 829 (2010) 176 [arXiv:0909.5226] [INSPIRE].

[18] X. Bekaert, E. Joung and J. Mourad, Effective action in a higher-spin background, JHEP 02 (2011) 048 [arXiv: 1012.2103] [INSPIRE]. 
[19] X. Bekaert and M. Grigoriev, Higher order singletons, partially massless fields and their boundary values in the ambient approach, Nucl. Phys. B 876 (2013) 667 [arXiv:1305.0162] [INSPIRE].

[20] R.R. Metsaev, Arbitrary spin conformal fields in (A)dS, Nucl. Phys. B 885 (2014) 734 [arXiv: 1404.3712] [INSPIRE].

[21] T. Nutma and M. Taronna, On conformal higher spin wave operators, JHEP 06 (2014) 066 [arXiv: 1404.7452] [INSPIRE].

[22] M. Beccaria, X. Bekaert and A.A. Tseytlin, Partition function of free conformal higher spin theory, JHEP 08 (2014) 113 [arXiv:1406.3542] [INSPIRE].

[23] M. Beccaria, S. Nakach and A.A. Tseytlin, On triviality of S-matrix in conformal higher spin theory, JHEP 09 (2016) 034 [arXiv: 1607.06379] [INSPIRE].

[24] M. Grigoriev and A.A. Tseytlin, On conformal higher spins in curved background, J. Phys. A 50 (2017) 125401 [arXiv: 1609.09381] [INSPIRE].

[25] S.M. Kuzenko, R. Manvelyan and S. Theisen, Off-shell superconformal higher spin multiplets in four dimensions, JHEP 07 (2017) 034 [arXiv: 1701.00682] [INSPIRE].

[26] M. Beccaria and A.A. Tseytlin, On induced action for conformal higher spins in curved background, Nucl. Phys. B 919 (2017) 359 [arXiv:1702.00222] [INSPIRE].

[27] R. Bonezzi, Induced Action for Conformal Higher Spins from Worldline Path Integrals, Universe 3 (2017) 64 [arXiv:1709.00850] [INSPIRE].

[28] R. Manvelyan and G. Poghosyan, Geometrical structure of Weyl invariants for spin three gauge field in general gravitational background in d=4, Nucl. Phys. B 937 (2018) 1 [arXiv: 1804.10779] [INSPIRE].

[29] T. Adamo, S. Nakach and A.A. Tseytlin, Scattering of conformal higher spin fields, JHEP 07 (2018) 016 [arXiv: 1805.00394] [INSPIRE].

[30] M. Grigoriev, I. Lovrekovic and E. Skvortsov, New Conformal Higher Spin Gravities in 3d, JHEP 01 (2020) 059 [arXiv:1909.13305] [INSPIRE].

[31] M. Grigoriev, K. Mkrtchyan and E. Skvortsov, Matter-free higher spin gravities in 3D: Partially-massless fields and general structure, Phys. Rev. D 102 (2020) 066003 [arXiv: 2005.05931] [INSPIRE].

[32] E.A. Bergshoeff, O. Hohm and P.K. Townsend, On Higher Derivatives in 3D Gravity and Higher Spin Gauge Theories, Annals Phys. 325 (2010) 1118 [arXiv:0911.3061] [INSPIRE].

[33] E.A. Bergshoeff, M. Kovacevic, J. Rosseel, P.K. Townsend and Y. Yin, A spin-4 analog of $3 D$ massive gravity, Class. Quant. Grav. 28 (2011) 245007 [arXiv:1109.0382] [INSPIRE].

[34] B.E.W. Nilsson, Towards an exact frame formulation of conformal higher spins in three dimensions, JHEP 09 (2015) 078 [arXiv: 1312.5883] [INSPIRE].

[35] B.E.W. Nilsson, On the conformal higher spin unfolded equation for a three-dimensional self-interacting scalar field, JHEP 08 (2016) 142 [arXiv:1506.03328] [INSPIRE].

[36] M. Henneaux, S. Hörtner and A. Leonard, Higher Spin Conformal Geometry in Three Dimensions and Prepotentials for Higher Spin Gauge Fields, JHEP 01 (2016) 073 [arXiv: 1511.07389] [INSPIRE].

[37] H. Linander and B.E.W. Nilsson, The non-linear coupled spin 2-spin 3 Cotton equation in three dimensions, JHEP 07 (2016) 024 [arXiv: 1602.01682] [INSPIRE]. 
[38] S.M. Kuzenko and D.X. Ogburn, Off-shell higher spin $N=2$ supermultiplets in three dimensions, Phys. Rev. D 94 (2016) 106010 [arXiv:1603.04668] [InSPIRE].

[39] S.M. Kuzenko, Higher spin super-Cotton tensors and generalisations of the linear-chiral duality in three dimensions, Phys. Lett. B 763 (2016) 308 [arXiv:1606.08624] [INSPIRE].

[40] S.M. Kuzenko and M. Tsulaia, Off-shell massive $N=1$ supermultiplets in three dimensions, Nucl. Phys. B 914 (2017) 160 [arXiv:1609.06910] [inSPIRE].

[41] T. Basile, R. Bonezzi and N. Boulanger, The Schouten tensor as a connection in the unfolding of $3 D$ conformal higher-spin fields, JHEP 04 (2017) 054 [arXiv:1701.08645] [INSPIRE].

[42] S.M. Kuzenko and M. Ponds, Topologically massive higher spin gauge theories, JHEP 10 (2018) 160 [arXiv: 1806.06643] [INSPIRE].

[43] M. Henneaux, V. Lekeu, A. Leonard, J. Matulich and S. Prohazka, Three-dimensional conformal geometry and prepotentials for four-dimensional fermionic higher-spin fields, JHEP 11 (2018) 156 [arXiv:1810.04457] [INSPIRE].

[44] E.I. Buchbinder, D. Hutchings, J. Hutomo and S.M. Kuzenko, Linearised actions for $\mathcal{N}$-extended (higher-spin) superconformal gravity, JHEP 08 (2019) 077 [arXiv:1905.12476] [INSPIRE].

[45] M.A. Vasiliev, Free Massless Fields of Arbitrary Spin in the de Sitter Space and Initial Data for a Higher Spin Superalgebra, Fortsch. Phys. 35 (1987) 741 [Yad. Fiz. 45 (1987) 1784] [INSPIRE].

[46] T. Damour and S. Deser, 'Geometry' of Spin 3 Gauge Theories, Ann. Inst. H. Poincaré Phys. Theor. 47 (1987) 277 [INSPIRE].

[47] R. Andringa, E.A. Bergshoeff, M. de Roo, O. Hohm, E. Sezgin and P.K. Townsend, Massive 3D Supergravity, Class. Quant. Grav. 27 (2010) 025010 [arXiv: 0907.4658] [inSPIRE].

[48] S. Deser and J.H. Kay, Topologically massive supergravity, Phys. Lett. B 120 (1983) 97 [INSPIRE].

[49] G.W. Gibbons, C.N. Pope and E. Sezgin, The General Supersymmetric Solution of Topologically Massive Supergravity, Class. Quant. Grav. 25 (2008) 205005 [arXiv: 0807.2613] [INSPIRE].

[50] N. Boulanger, D. Ponomarev, E. Sezgin and P. Sundell, New unfolded higher spin systems in $A d S_{3}$, Class. Quant. Grav. 32 (2015) 155002 [arXiv: 1412.8209] [INSPIRE].

[51] E.A. Bergshoeff, O. Hohm, J. Rosseel, E. Sezgin and P.K. Townsend, On Critical Massive (Super)Gravity in $A d S_{3}$, J. Phys. Conf. Ser. 314 (2011) 012009 [arXiv:1011.1153] [INSPIRE].

[52] I.V. Gorbunov, S.M. Kuzenko and S.L. Lyakhovich, On the minimal model of anyons, Int. J. Mod. Phys. A 12 (1997) 4199 [hep-th/9607114] [INSPIRE].

[53] I.V. Tyutin and M.A. Vasiliev, Lagrangian formulation of irreducible massive fields of arbitrary spin in $(2+1)$-dimensions, Teor. Mat. Fiz. 113N1 (1997) 45 [Theor. Math. Phys. 113 (1997) 1244] [hep-th/9704132] [INSPIRE].

[54] S. Deser and R.I. Nepomechie, Anomalous Propagation of Gauge Fields in Conformally Flat Spaces, Phys. Lett. B 132 (1983) 321 [inSPIRE]. 
[55] A. Higuchi, Forbidden Mass Range for Spin-2 Field Theory in de Sitter Space-time, Nucl. Phys. B 282 (1987) 397 [INSPIRE].

[56] A. Higuchi, Symmetric Tensor Spherical Harmonics on the $N$ Sphere and Their Application to the de Sitter Group $\mathrm{SO}(N, 1)$, J. Math. Phys. 28 (1987) 1553 [Erratum ibid. 43 (2002) 6385] [INSPIRE].

[57] L. Brink, R.R. Metsaev and M.A. Vasiliev, How massless are massless fields in AdS $S_{d}$, Nucl. Phys. B 586 (2000) 183 [hep-th/0005136] [InSPIRE].

[58] S. Deser and A. Waldron, Gauge invariances and phases of massive higher spins in $(A) d S$, Phys. Rev. Lett. 87 (2001) 031601 [hep-th/0102166] [INSPIRE].

[59] S. Deser and A. Waldron, Partial masslessness of higher spins in $(A) d S$, Nucl. Phys. B 607 (2001) 577 [hep-th/0103198] [inSPIRE].

[60] S. Deser and A. Waldron, Null propagation of partially massless higher spins in $(A) d S$ and cosmological constant speculations, Phys. Lett. B 513 (2001) 137 [hep-th/0105181] [INSPIRE].

[61] L. Dolan, C.R. Nappi and E. Witten, Conformal operators for partially massless states, JHEP 10 (2001) 016 [hep-th/0109096] [INSPIRE].

[62] Y.M. Zinoviev, On massive high spin particles in AdS, hep-th/0108192 [INSPIRE].

[63] R.R. Metsaev, Gauge invariant formulation of massive totally symmetric fermionic fields in (A)dS space, Phys. Lett. B 643 (2006) 205 [hep-th/0609029] [INSPIRE].

[64] E.D. Skvortsov and M.A. Vasiliev, Geometric formulation for partially massless fields, Nucl. Phys. B 756 (2006) 117 [hep-th/0601095] [INSPIRE].

[65] I.L. Buchbinder, T.V. Snegirev and Y.M. Zinoviev, Gauge invariant Lagrangian formulation of massive higher spin fields in $(A) d S_{3}$ space, Phys. Lett. B 716 (2012) 243 [arXiv: 1207.1215] [INSPIRE].

[66] I.L. Buchbinder, T.V. Snegirev and Y.M. Zinoviev, Frame-like gauge invariant Lagrangian formulation of massive fermionic higher spin fields in $A d S_{3}$ space, Phys. Lett. B 738 (2014) 258 [arXiv: 1407.3918] [INSPIRE].

[67] S. Deger, A. Kaya, E. Sezgin and P. Sundell, Spectrum of $D=6, N=4 b$ supergravity on $A d S_{3} \times S^{3}$, Nucl. Phys. B 536 (1998) 110 [hep-th/9804166] [INSPIRE].

[68] S. Deser and A. Waldron, Arbitrary spin representations in de Sitter from dS/CFT with applications to $d S$ supergravity, Nucl. Phys. B 662 (2003) 379 [hep-th/0301068] [INSPIRE].

[69] S. Deser, R. Jackiw and S. Templeton, Three-Dimensional Massive Gauge Theories, Phys. Rev. Lett. 48 (1982) 975 [INSPIRE].

[70] S. Deser, R. Jackiw and S. Templeton, Topologically Massive Gauge Theories, Annals Phys. 140 (1982) 372 [Erratum ibid. 185 (1988) 406] [INSPIRE].

[71] C. Fronsdal, Singletons and Massless, Integral Spin Fields on de Sitter Space (Elementary Particles in a Curved Space. 7, Phys. Rev. D 20 (1979) 848 [InSPIRE].

[72] J. Fang and C. Fronsdal, Massless, Half Integer Spin Fields in de Sitter Space, Phys. Rev. D 22 (1980) 1361 [INSPIRE].

[73] D. Hutchings, J. Hutomo and S.M. Kuzenko, Higher-spin gauge models with $(1,1)$ supersymmetry in $A d S_{3}$ : Reduction to $(1,0)$ superspace, arXiv:2011.14294 [INSPIRE]. 
[74] S.M. Kuzenko and G. Tartaglino-Mazzucchelli, Conformal supergravities as Chern-Simons theories revisited, JHEP 03 (2013) 113 [arXiv:1212.6852] [INSPIRE].

[75] B.M. Zupnik and D.G. Pak, Superfield Formulation of the Simplest Three-dimensional Gauge Theories and Conformal Supergravities, Theor. Math. Phys. 77 (1988) 1070 [Teor. Mat. Fiz. 77 (1988) 97] [INSPIRE].

[76] S.M. Kuzenko, Prepotentials for $N=2$ conformal supergravity in three dimensions, JHEP 12 (2012) 021 [arXiv: 1209.3894] [INSPIRE].

[77] S.M. Kuzenko, U. Lindström and G. Tartaglino-Mazzucchelli, Off-shell supergravity-matter couplings in three dimensions, JHEP 03 (2011) 120 [arXiv:1101.4013] [INSPIRE].

[78] P.S. Howe, J.M. Izquierdo, G. Papadopoulos and P.K. Townsend, New supergravities with central charges and Killing spinors in (2+1)-dimensions, Nucl. Phys. B 467 (1996) 183 [hep-th/9505032] [INSPIRE].

[79] S.M. Kuzenko, J. Novak and G. Tartaglino-Mazzucchelli, $N=6$ superconformal gravity in three dimensions from superspace, JHEP 01 (2014) 121 [arXiv: 1308.5552] [INSPIRE].

[80] D. Butter, S.M. Kuzenko, J. Novak and G. Tartaglino-Mazzucchelli, Conformal supergravity in three dimensions: Off-shell actions, JHEP 10 (2013) 073 [arXiv: 1306.1205] [INSPIRE].

[81] P. van Nieuwenhuizen, D = 3 Conformal Supergravity and Chern-Simons Terms, Phys. Rev. D 32 (1985) 872 [INSPIRE].

[82] M. Roček and P. van Nieuwenhuizen, $N \geq 2$ supersymmetric Chern-Simons terms as $D=3$ extended conformal supergravity, Class. Quant. Grav. 3 (1986) 43 [INSPIRE].

[83] M. Nishimura and Y. Tanii, $N=6$ conformal supergravity in three dimensions, JHEP 10 (2013) 123 [arXiv: 1308.3960] [INSPIRE].

[84] U. Lindström and M. Roček, Superconformal Gravity in Three-dimensions as a Gauge Theory, Phys. Rev. Lett. 62 (1989) 2905 [InSPIRE].

[85] H. Nishino and S.J. Gates Jr., Chern-Simons theories with supersymmetries in three-dimensions, Int. J. Mod. Phys. A 8 (1993) 3371 [inSPIRE].

[86] S.J. Gates Jr., M.T. Grisaru, M. Roček and W. Siegel, Superspace Or One Thousand and One Lessons in Supersymmetry, Front. Phys. 58 (1983) 1 [hep-th/0108200] [INSPIRE].

[87] B.M. Zupnik and D.G. Pak, Differential and Integral Forms in Supergauge Theories and Supergravity, Class. Quant. Grav. 6 (1989) 723 [InSPIRE].

[88] U. Lindström and M. Roček, A super-Weyl-invariant spinning membrane, Phys. Lett. B 218 (1989) 207 [Conf. Proc. C 8903131 (1989) 341] [InSPIRE].

[89] S.M. Kuzenko, U. Lindström and G. Tartaglino-Mazzucchelli, Three-dimensional $(p, q)$ AdS superspaces and matter couplings, JHEP 08 (2012) 024 [arXiv: 1205.4622] [INSPIRE].

[90] S.M. Kuzenko, J. Novak and G. Tartaglino-Mazzucchelli, Higher derivative couplings and massive supergravity in three dimensions, JHEP 09 (2015) 081 [arXiv:1506.09063] [INSPIRE].

[91] I.L. Buchbinder, T.V. Snegirev and Y.M. Zinoviev, Lagrangian formulation of the massive higher spin supermultiplets in three dimensional space-time, JHEP 10 (2015) 148 [arXiv: 1508.02829] [INSPIRE].

[92] I.L. Buchbinder, T.V. Snegirev and Y.M. Zinoviev, Lagrangian description of massive higher spin supermultiplets in $A d S_{3}$ space, JHEP 08 (2017) 021 [arXiv:1705.06163] [INSPIRE]. 
[93] I.L. Buchbinder, T.V. Snegirev and Y.M. Zinoviev, Supersymmetric higher spin models in three dimensional spaces, Symmetry 10 (2017) 9 [arXiv:1711.11450] [INSPIRE].

[94] Y.M. Zinoviev, Frame-like gauge invariant formulation for massive high spin particles, Nucl. Phys. B 808 (2009) 185 [arXiv:0808.1778] [inSPIRE].

[95] A.A. Tseytlin, Effective action in de Sitter space and conformal supergravity (in Russian), Yad. Fiz. 39 (1984) 1606 [Sov. J. Nucl. Phys. 39 (1984) 1018] [inSPIRE].

[96] E.S. Fradkin and A.A. Tseytlin, Instanton zero modes and $\beta$-functions in supergravities. 2. Conformal supergravity, Phys. Lett. B 134 (1984) 307 [INSPIRE].

[97] E. Joung and K. Mkrtchyan, A note on higher-derivative actions for free higher-spin fields, JHEP 11 (2012) 153 [arXiv:1209.4864] [INSPIRE].

[98] A.A. Tseytlin, On partition function and Weyl anomaly of conformal higher spin fields, Nucl. Phys. B 877 (2013) 598 [arXiv:1309.0785] [INSPIRE].

[99] E. Joung and K. Mkrtchyan, Weyl Action of Two-Column Mixed-Symmetry Field and Its Factorization Around (A)dS Space, JHEP 06 (2016) 135 [arXiv: 1604.05330] [INSPIRE].

[100] M. Grigoriev and A. Hancharuk, On the structure of the conformal higher-spin wave operators, JHEP 12 (2018) 033 [arXiv:1808.04320] [INSPIRE].

[101] S.M. Kuzenko and M. Ponds, Spin projection operators in $(A) d S$ and partial masslessness, Phys. Lett. B 800 (2020) 135128 [arXiv:1910.10440] [INSPIRE].

[102] E.I. Buchbinder, S.M. Kuzenko, J. La Fontaine and M. Ponds, Spin projection operators and higher-spin Cotton tensors in three dimensions, Phys. Lett. B 790 (2019) 389 [arXiv: 1812.05331] [INSPIRE].

[103] E.I. Buchbinder, D. Hutchings, S.M. Kuzenko and M. Ponds, AdS superprojectors, JHEP 04 (2021) 074 [arXiv: 2101.05524] [InSPIRE]. 